\title{
Orbital and spectral analysis of the benchmark brown dwarf HD 4747B ${ }^{\star}$
}

S. Peretti ${ }^{1}$, D. Ségransan ${ }^{1}$, B. Lavie ${ }^{1}$, S. Desidera ${ }^{2}$, A.-L. Maire ${ }^{3}$, V. D’Orazi ${ }^{2}$, A. Vigan ${ }^{4}$, J.-L. Baudino ${ }^{5,6}$, A. Cheetham ${ }^{1}$, M. Janson ${ }^{3,7}$, G. Chauvin ${ }^{8}$, J. Hagelberg ${ }^{8}$, F. Menard ${ }^{8}$, Kevin Heng ${ }^{9}$, S. Udry ${ }^{1}$, A. Boccaletti ${ }^{5}$, S. Daemgen ${ }^{11}$, H. Le Coroller ${ }^{4}$, D. Mesa ${ }^{2}$, D. Rouan ${ }^{5}$, M. Samland ${ }^{3}$, T. Schmidt ${ }^{5}$, A. Zurlo ${ }^{4,17,18}$, M. Bonnefoy ${ }^{8}$, M. Feldt ${ }^{3}$, R. Gratton ${ }^{2}$, A.-M. Lagrange ${ }^{8}$, M. Langlois ${ }^{4,13}$, M. Meyer ${ }^{11,14}$, M. Carbillet ${ }^{10}$, M. Carle ${ }^{4}$, V. De Caprio ${ }^{12}$, L. Gluck ${ }^{8}$, E. Hugot ${ }^{4}$, Y. Magnard ${ }^{8}$, T. Moulin ${ }^{8}$, A. Pavlov ${ }^{3}$, J. Pragt ${ }^{15}$, P. Rabou ${ }^{8}$, J. Ramos ${ }^{3}$, G. Rousset ${ }^{5}$, A. Sevin ${ }^{5}$, C. Soenke ${ }^{16}$, E. Stadler ${ }^{8}$, L. Weber ${ }^{1}$, and F. Wildi ${ }^{1}$

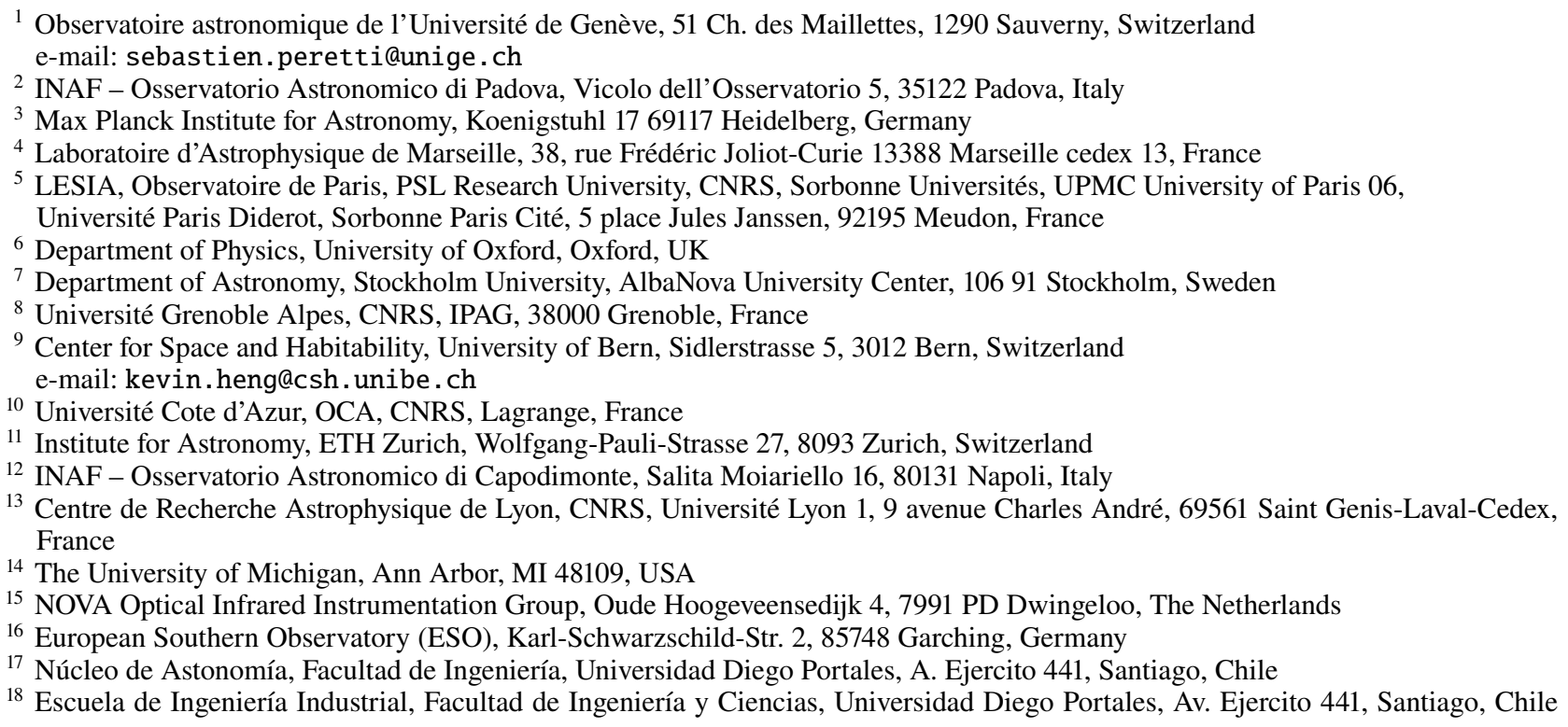

Received 12 December 2017 / Accepted 13 May 2018

\section{ABSTRACT}

Context. The study of high-contrast imaged brown dwarfs and exoplanets depends strongly on evolutionary models. To estimate the mass of a directly imaged substellar object, its extracted photometry or spectrum is used and adjusted with model spectra together with the estimated age of the system. These models still need to be properly tested and constrained. HD 4747B is a brown dwarf close to the $\mathrm{H}$ burning mass limit, orbiting a nearby $(d=19.25 \pm 0.58 \mathrm{pc})$, solar-type star $(\mathrm{G} 9 \mathrm{~V})$; it has been observed with the radial velocity method for over almost two decades. Its companion was also recently detected by direct imaging, allowing a complete study of this particular object.

Aims. We aim to fully characterize HD 4747B by combining a well-constrained dynamical mass and a study of its observed spectral features in order to test evolutionary models for substellar objects and to characterize its atmosphere.

Methods. We combined the radial velocity measurements of High Resolution Echelle Spectrometer (HIRES) and CORALIE taken over two decades and high-contrast imaging of several epochs from NACO, NIRC2, and SPHERE to obtain a dynamical mass. From the SPHERE data we obtained a low-resolution spectrum of the companion from $Y$ to $H$ band, and two narrow band-width photometric measurements in the $K$ band. A study of the primary star also allowed us to constrain the age of the system and its distance.

Results. Thanks to the new SPHERE epoch and NACO archival data combined with previous imaging data and high-precision radial velocity measurements, we were able to derive a well-constrained orbit. The high eccentricity $(e=0.7362 \pm 0.0025)$ of HD $4747 \mathrm{~B}$ is confirmed, and the inclination and the semi-major axis are derived $\left(i=47.3 \pm 1.6^{\circ}, a=10.01 \pm 0.21 \mathrm{au}\right)$. We derive a dynamical mass of $m_{\mathrm{B}}=70.0 \pm 1.6 M_{\mathrm{Jup}}$, which is higher than a previous study but in better agreement with the models. By comparing the object with known brown dwarfs spectra, we derive a spectral type of L9 and an effective temperature of $1350 \pm 50 \mathrm{~K}$. With a retrieval analysis we constrain the oxygen and carbon abundances and compare them with the values from the HR 8799 planets.

Key words. binaries: general - binaries: spectroscopic - binaries: visual - brown dwarfs - planets and satellites: atmospheres techniques: high angular resolution

\footnotetext{
^ Based on observations made with the instrument SPHERE (Prog. ID 198.C-0209) and NaCo (Prog. ID 081.C-0917(A)) at the Paranal observatory and with the CORALIE echelle spectrograph mounted on the $1.2 \mathrm{~m}$ Swiss telescope at La Silla Observatory.
} 


\section{Introduction}

Brown dwarfs (BDs) are substellar objects, not massive enough to sustain hydrogen burning, with masses below $\sim 75$ Jupiter masses (e.g., Burrows et al. 1997). Since the first brown dwarfs were discovered by imaging in 1995 (Nakajima et al. 1995; Oppenheimer et al. 1995; Rebolo et al. 1995), only a few of these objects have been detected around sun-like stars with respect to the number of planetary mass objects, and binaries (e.g., Anderson et al. 2011; Sahlmann et al. 2011a; Siverd et al. 2012; Bayliss et al. 2017). Numerous studies based on transiting, radial velocity and astrometry methods have indeed demonstrated that the "brown dwarf desert" is observed at separations below 10 au and that brown dwarfs have a frequency around sun-like stars of less than 1\% (Halbwachs et al. 2000; Marcy \& Butler 2000; Grether \& Lineweaver 2006; Sahlmann et al. 2011b; Wilson et al. 2016). However, a recent study showed that this desert might exist only for separations smaller than $\sim 0.1-\sim 0.2$ au (e.g., Troup et al. 2016). At larger separations, BD are found as abundantly as very low-mass stars. However, the much wider range in spectral types in comparison with radial velocity (RV) surveys probed by Troup et al. (2016) might explain the high number of BDs found.

Due to observing biases, direct imaging is much better suited to exploring the outer reaches of stellar systems to search for brown dwarf companions. A few brown dwarfs have been detected by direct imaging (e.g., Thalmann et al. 2009; Biller et al. 2010; Chauvin et al. 2010), allowing us to constrain their effective temperature and atmospheric properties thanks to spectrophotometry analysis (e.g., Maire et al. 2016a; Vigan et al. 2016) and the age of the system through the host stars. To determine the mass of an imaged BD companion, the key parameter for the evolution of substellar objects, we usually rely on evolutionary models (e.g., Baraffe et al. 2003). These models still need to be tested and properly calibrated through observations. To achieve this, observations of objects for which we can independently constrain the age, the effective temperature, and the mass are needed.

Free floating BDs have been detected and they have provided high-resolution spectra, allowing us to better understand these objects (see, e.g., Kirkpatrick 2005; Helling \& Casewell 2014, and references therein), but independent mass and age estimations cannot be derived in most cases. BD companions to solar-type stars have been discovered at wide orbits (e.g., Burgasser 2007b; Pinfield et al. 2012; Burningham et al. 2013, and references therein), allowing an age determination from their host stars and effective temperature from their spectra. However, their wide orbits prevent a dynamical mass measurement. Dynamical masses of BDs have been determined for BD pairs (e.g., Dupuy \& Liu 2017), but no independent age estimation could be extracted for these substellar systems. For some objects we have high-resolution spectra and dynamical masses, but they often also lack independent age measurements (e.g., King et al. 2010; Line et al. 2015). The majority of directly imaged brown dwarf companions from high-contrast imaging surveys have been detected around young and massive stars as they are brighter at young ages (Chabrier \& Baraffe 2000). These detections can provide age estimations of the system from the stellar host, and from astrometric orbits, but model-independent dynamical masses cannot be easily obtained due to the difficulties in achieving precise radial velocities of young, massive stars (e.g., Galland et al. 2005). In addition, the orbital period of these directly imaged brown dwarfs are usually on timescales of decades if not centuries, and therefore obtaining a complete orbit will need long-term monitoring.

HD 4747B is a perfect candidate for testing the evolutionary models. It is orbiting a late G-type star (HD 4747), which was observed through two decades by the HIRES instrument at the Keck (Vogt et al. 1994), and with the CORALIE spectrograph (Queloz et al. 2000) to obtain radial velocities. Two-thirds of the orbit has been already completed, and the important periastron passage has been covered. This has allowed a minimum mass determination of the companion that pointed towards a brown dwarf at large separation (Nidever et al. 2002; Sahlmann et al. $2011 \mathrm{~b})$. Thanks to the high precision of the RV data and the number of points, the minimum mass is well constrained and only a few astrometric points can provide a high-precision orbit and dynamical mass. HD 4747B was directly imaged for the first time by Crepp et al. (2016) who confirmed its substellar nature and gave a dynamical mass estimation of $m_{\mathrm{B}}=60.2 \pm 3.3 \mathrm{M}_{\mathrm{Jup}}{ }^{1}$. However the isochronal mass estimate of $m_{\mathrm{B}}=72_{-13}^{+3} M_{\mathrm{Jup}}$ was in marginal agreement with its dynamical mass. A color-magnitude diagram led to a late $\mathrm{L}$ spectral type.

To improve the orbital parameters and characterize the atmosphere of HD 4747B, we observed HD 4747 with the SPHERE instrument installed on the VLT (Spectro-Polarimetric Highcontrast Exoplanet REsearch; Beuzit et al. 2008) in December 2016. In Sect. 2 we detail our analysis of the host star, which gives the age and distance of the system. We describe in Sect. 3 the observations used in this paper. In Sect. 4 we report on the extraction of spectrophotometry and astrometry from the SPHERE images and we also describe the analysis of an archival NACO dataset in which we were able to detect the companion. Section 5 presents our orbital analysis combining radial velocity and imaging data. Section 6 describes the spectrophotometric analysis by comparing our extracted spectra with real objects, models, and a retrieval analysis. We conclude in Sect. 7.

\section{Host star properties}

As a close ( $d=19.3 \pm 0.6 \mathrm{pc}$; this paper $)$ solar-type star, HD 4747 has been extensively studied in the literature. Several spectroscopic analyses were performed by different groups (Table 1), indicating an effective temperature around 5300-5400 K, a gravity $\log g=4.5-4.65$ and a mildly subsolar metallicity $([\mathrm{Fe} / \mathrm{H}]$ about -0.2). Similar values were also derived from Strömgren photometry.

We used an archival spectrum of HD 4747 taken with the visible high-resolution spectrograph FEROS $^{2}$ (Kaufer et al. 1997), to rederive its stellar parameters. The effective temperature $\left(T_{\text {eff }}\right)$, the gravity $(\log g)$, the microturbulence $(\xi)$, and the metallicity $([\mathrm{Fe} / \mathrm{H}])$ were retrieved using the standard approach described in D'Orazi et al. (2017). We obtain $T_{\text {eff }}=5400 \pm 60 \mathrm{~K}, \log g=4.60 \pm 0.15 \mathrm{dex}, \xi=0.75 \pm 0.2 \mathrm{~km} \mathrm{~s}^{-1}$, and $[\mathrm{Fe} / \mathrm{H}]=-0.23 \pm 0.05 \mathrm{dex}$. Our results, also reported in Table 1, are fully consistent with those from the literature. Moreover, we derived abundances for elements produced in the slow $(s)$ neutron-capture process, namely yttrium, barium, and lanthanum. As was done in all our previous investigations, we carried out spectral synthesis calculations, including isotopic splitting and hyperfine structure as needed (see, e.g., D'Orazi et al. 2012, 2017). We detected a modest enhancement

1 During the referee process of this paper, additional results from observations taken with GPI were published by Crepp et al. (2018). The dynamical mass has been updated at $m_{\mathrm{B}}=65.3_{-3.3}^{+4.4} M_{\mathrm{Jup}}$.

2 PI: Rolf Chini, Prog. ID 095.A-9029. 
Table 1. Spectroscopic parameters of HD 4747.

\begin{tabular}{llllll}
\hline \hline$T_{\text {eff }}[\mathrm{K}]$ & $\log g[\mathrm{dex}]$ & $\xi\left[\mathrm{km} \mathrm{s}^{-1}\right]$ & {$[\mathrm{Fe} / \mathrm{H}][\mathrm{dex}]$} & $v \sin i\left[\mathrm{~km} \mathrm{~s}^{-1}\right]$ & Reference \\
\hline $5337 \pm 80$ & $4.58 \pm 0.10$ & $0.85 \pm 0.20$ & $-0.25 \pm 0.07$ & $2.3 \pm 1.0$ & Fuhrmann et al. (2017) \\
5347 & & -0.21 & & Mortier et al. (2013) \\
$5422 \pm 75$ & 4.61 & & -0.15 & & Casagrande et al. (2011) \\
5305 & 4.56 & -0.24 & 2.1 & Brewer et al. (2016) \\
5335 & 4.65 & & -0.22 & 1.1 & Valenti \& Fischer (2005) \\
$5316 \pm 38$ & $4.48 \pm 0.10$ & $0.79 \pm 0.06$ & $-0.21 \pm 0.05$ & 0.79 & Santos et al. (2005) \\
$5340 \pm 40$ & $4.65 \pm 0.06$ & & $-0.22 \pm 0.04$ & $1.1 \pm 0.5$ & Crepp et al. (2016) \\
\hline $5400 \pm 60$ & $4.60 \pm 0.15$ & $0.75 \pm 0.20$ & $-0.23 \pm 0.05$ & $2.0 \pm 1.0$ & This paper \\
\hline
\end{tabular}

in $s$-process element abundances, finding $[\mathrm{Y} / \mathrm{Fe}]=+0.30 \pm 0.15$, $[\mathrm{Ba} / \mathrm{Fe}]=+0.35 \pm 0.20 \mathrm{dex}$ and $[\mathrm{La} / \mathrm{Fe}]=+0.20 \pm 0.12 \mathrm{dex}$. This might be in principle the signature of a weak contamination from a companion during the asymptotic giant branch (AGB) phase, but no indication of the presence of a white dwarf companion at any separation was detected and the HD 4747B spectrum is not compatible with a white dwarf spectrum. Most importantly, we also derived carbon abundance (pollution from low-mass AGB stars results in enhanced $\mathrm{C}$ abundances) by synthesizing the $\mathrm{CH}$ band at $4300 \AA$. We obtain a solar-scaled abundance of $[\mathrm{C} / \mathrm{Fe}]=0 \pm 0.13 \mathrm{dex}$, which goes against the AGB pollution scenario. Moreover, when observation uncertainties are taken into account, the $s$ process element abundances for HD 4747 are consistent with the scattered distribution, as revealed from field stars (see, e.g., Bensby et al. 2014).

A crucial piece of information for the characterization of the low-mass companion HD 4747B is the stellar age. The literature shows quite discrepant values due to the different dating techniques employed. As expected for a late G/early $\mathrm{K}$ dwarf close to the main sequence, isochrone fitting allows only a poor constraint on stellar age (basically upper limits of about 7-9 Gyr). The spectrum shows no lithium, putting a lower limit to stellar age at about $700 \mathrm{Myr}$.

The best constraints are derived from coronal and chromospheric emission. Using the Keck $S$ index values reported in Butler et al. (2017) and $B-V$ from Hipparcos, we derived a median values of $\log R_{\mathrm{HK}}=-4.725$ with a rms of 0.023 dex. From the CORALIE dataset described in Sect. 3.1, $\log R_{\mathrm{HK}}=$ -4.718 is obtained, with a rms of 0.007 dex (see Fig. 1).

These values are intermediate between the activity levels of the Hyades and M 67 open clusters and clearly above that of the Sun. The extension of the Keck dataset (50 epochs over almost $18 \mathrm{yr}$ ) ensures that intrinsic variability is averaged out. Using the age- $\log R_{\mathrm{HK}}$ calibration by Mamajek \& Hillenbrand (2008) an age of $2.3 \mathrm{Gyr}$ is obtained. A very similar age, $2.1 \mathrm{Gyr}$, is obtained from X-ray emission (Katsova \& Livshits 2011), when applying Mamajek \& Hillenbrand (2008) calibration. On the basis of these results we favor an age of $2.3 \mathrm{Gyr}$ with upper and lower limits of 0.9-3.7 Gyr as the activity level of HD 4747 is clearly distinct from the Hyades members' activities at the young side and from the Sun's activity even at its maximum of the activity cycle at the old side. We also used the Mamajek \& Hillenbrand (2008) calibration in order to compute the expected rotational period of HD 4747 and found $P_{\text {rot }}=25.8 \pm 4.1$ days .

By looking at the periodograms of the $\log R_{\mathrm{HK}}$ (Fig. 2a) we find a clear signal at 27.7 days. A signal is observed at the same period in the periodogram as the residual of the radial velocity data (Fig. 2b). We therefore favor a rotational period of $P_{\text {rot }}=$ $27.7 \pm 0.5$ days.

Fuhrmann et al. (2017) noted that the kinematic parameters support membership in the Hyades stream, and also the much

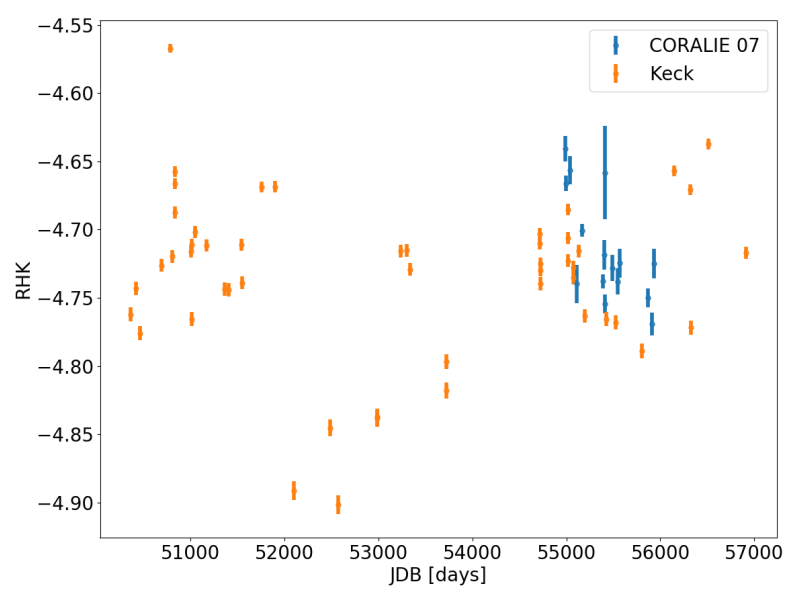

Fig. 1. $\log \left(R_{\mathrm{HK}}\right)$ from the Keck and CORALIE data.

lower metallicity of HD 4747 with respect to the Hyades. They also mention a possible inconsistency between the low metallicity and the activity level (moderately young age), and speculated about the possibility of accretion of angular momentum making the star appear younger (see D'Orazi et al. 2017, for a description of this mechanism in the case of GJ504). Conclusive proof that this mechanism is really at work in the case of HD 4747 is very challenging to obtain with respect to GJ504 because of the different main sequence lifetimes. However, to shed further light on this possibility, we investigated whether the age/metallicity obtained for HD 4747 is really peculiar using the extensive database of the Geneva-Copenhagen Survey (Nordström et al. 2004). Exploiting the latest age and metallicity determination by Casagrande et al. (2011), we searched for stars with ages within our adopted upper limit for HD 4747 and metallicity within \pm 0.05 dex. Hundreds of objects were returned, typically F-type stars, indicating that the age/metallicity combination of HD 4747 is not particularly unusual. Extended moving groups like the Hyades stream were also shown to host a mixture of stellar populations (Famaey et al. 2005), which means that HD 4747 is not particularly anomalous even from this point of view. While accretion events altering the angular momentum evolution and the age from activity cannot be firmly ruled out, we thus conclude that there are no specific indications supporting that this kind of evolution affected our target. Therefore, we dismiss this hypothesis and we adopt in the following the age from coronal and chromospheric activity.

The stellar mass was derived from isochrones using the PARAM web interface ${ }^{4}$ (da Silva et al. 2006) isolating the age

\footnotetext{
3 The graphs were prepared using a set of online tools hosted by the Data \& Analysis Center for Exoplanets (DACE), which is available at http://dace.unige.ch
}

4 http://stev.oapd.inaf.it/cgi-bin/param_1.3 


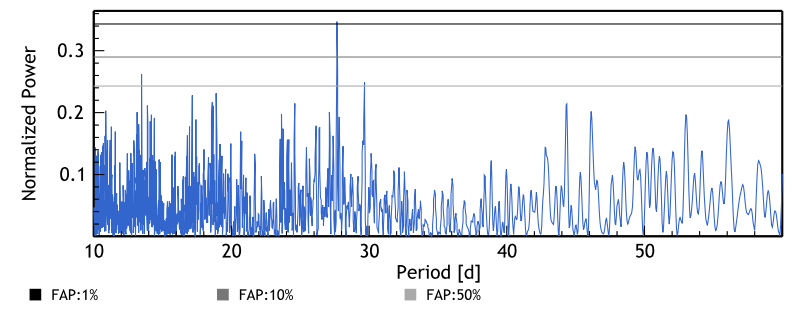

(a)

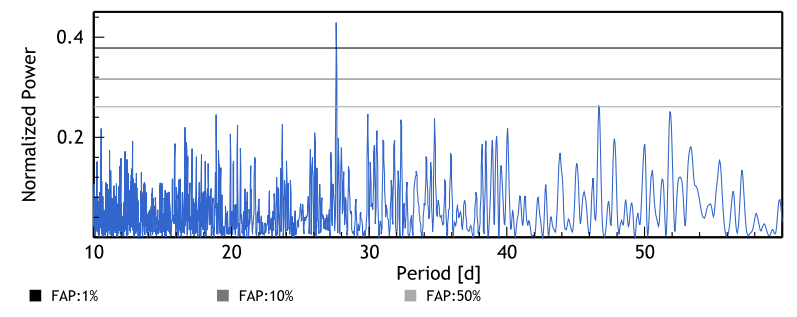

(b)

Fig. 2. GLS periodogram (Zechmeister \& Kürster 2009) of the $\log R_{\mathrm{HK}}$ (panel $a$ ) and the residual of the radial velocity data (panel $b$ ) from the HIRES and CORALIE spectrographs (see Sect. 5 for the orbital parameters) $)^{3}$.

range allowed by indirect methods (see Desidera et al. 2015). Our spectroscopic $T_{\text {eff }}$ and $[\mathrm{Fe} / \mathrm{H}]$ were adopted. The outcome is slightly dependent on the adopted distance. The HIPPARCOS and Gaia DR 1 distance are formally discrepant at more than a $4 \sigma$ level, possibly owing to the unaccounted orbital motion due to the brown dwarf companion ${ }^{5}$. Adopting the average of the two measurements, the stellar mass is $0.856 \pm 0.014 M_{\odot}$.

Finally, the star was observed with Spitzer and Herschel, resulting in no detectable IR excess (Gáspár et al. 2013). The stellar parameters are summarized in Table 2.

\section{Observations}

Radial velocity and direct imaging observations were combined to constrain the orbit of HD 4747B. The good orbital coverage of the radial velocity time series allows us to constrain HD 4747B's period, minimum mass, eccentricity, argument of periastron $\omega$, and time of periastron passage $T_{0}$. Combined with the few direct imaging observations spread over $\sim 27 \%$ of the period we were able to retrieve both longitude of ascending node $\Omega$ and the orbit inclination $i$. In addition, the SPHERE direct imaging observations allowed us to obtain a spectrum of the brown dwarf companion.

\subsection{Radial velocity}

HD 4747 has been observed since 1999 with the CORALIE spectrograph (Queloz et al. 2000) installed on the $1.2 \mathrm{~m}$ EULER Swiss telescope at La Silla observatory (Chile). Since its installation in 1998, CORALIE has undergone two upgrades (in 2007 and 2014) that introduced small RV offsets that vary from star to star. In the RV modeling procedure, we adjust the RV offsets corresponding to C98 for the data prior to 2007, to C07 for the period between 2007-2014, and C14 for the data acquired since 2014.

We combined these data with observations conducted between 1996 and 2014 (Butler et al. 2017), with the High Resolution Echelle Spectrometer (HIRES) at Keck (Vogt et al. 1994).

\footnotetext{
5 This is also supported by the formally significant differences in proper motion in various catalogs.
}

Table 2. Stellar parameters of HD 4747.

\begin{tabular}{|c|c|c|}
\hline Parameter & Value & Ref. \\
\hline$V(\mathrm{mag})$ & 7.15 & HIPPARCOS \\
\hline$B-V(\mathrm{mag})$ & $0.769 \pm 0.009$ & HIPPARCOS \\
\hline$V-I(\mathrm{mag})$ & $0.82 \pm 0.02$ & HIPPARCOS \\
\hline$J(\mathrm{mag})$ & $5.813 \pm 0.021$ & 2MASS \\
\hline$H$ (mag) & $5.433 \pm 0.049$ & 2MASS \\
\hline$K(\mathrm{mag})$ & $5.305 \pm 0.029$ & 2MASS \\
\hline Parallax (mas) & $53.51 \pm 0.53$ & van Leeuwen (2007) \\
\hline Parallax (mas) & $50.37 \pm 0.55$ & Gaia Collaboration (2016) \\
\hline Parallax (mas) & $51.94 \pm 1.57$ & Adopted for this paper \\
\hline$\mu_{\alpha}\left(\operatorname{mas~yr}^{-1}\right)$ & $516.92 \pm 0.55$ & van Leeuwen (2007) \\
\hline$\mu_{\delta}\left(\operatorname{mas~yr}^{-1}\right)$ & $120.05 \pm 0.45$ & van Leeuwen (2007) \\
\hline$\mu_{\alpha}\left(\operatorname{mas~yr}^{-1}\right)$ & $515.509 \pm 0.032$ & Gaia Collaboration (2016) \\
\hline$\mu_{\delta}\left(\operatorname{mas~yr}^{-1}\right)$ & $125.472 \pm 0.031$ & Gaia Collaboration (2016) \\
\hline$T_{\text {eff }}(\mathrm{K})$ & $5400 \pm 60$ & This paper \\
\hline $\log g$ & $4.60 \pm 0.15$ & This paper \\
\hline$[\mathrm{Fe} / \mathrm{H}]$ & $-0.23 \pm 0.05$ & This paper \\
\hline$v \sin i\left(\mathrm{~km} \mathrm{~s}^{-1}\right)$ & $2 \pm 1$ & This paper \\
\hline $\log R_{\mathrm{HK}}$ & -4.725 & Keck \\
\hline $\log R_{\mathrm{HK}}$ & -4.718 & CORALIE \\
\hline$P_{\text {rot }}$ (days) & $27.7 \pm 0.5$ & This paper \\
\hline $\log L_{\mathrm{X}} / L_{\mathrm{bol}}$ & -5.52 & Katsova \& Livshits (2011) \\
\hline 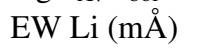 & 0 & This paper \\
\hline Age (Gyr) & $2.3 \pm 1.4$ & This paper \\
\hline$M_{\text {star }}\left(M_{\odot}\right)$ & $0.856 \pm 0.014$ & This paper \\
\hline$R_{\text {star }}\left(R_{\odot}\right)$ & $0.769 \pm 0.016$ & This paper \\
\hline
\end{tabular}

All of the observations were taken with high signal-to-noise ratios $(\mathrm{S} / \mathrm{N})$, thanks to the relative brightness of the primary star $(V=7.155)$. The high eccentricity $(e=0.736 \pm 0.002)$ of the companion and the fact that we have the periastron passage with the HIRES observations, together with the very long time span $(20 \mathrm{yr})$ and the high precision of the data allow us to constrain very well the orbital elements, even if only $57 \%$ of the complete orbit is covered.

\subsection{Direct imaging}

HD 4747 was observed on 12 December 2016 and 28 of September 2017 as part of the SpHere INfrared survey for Exoplanets (SHINE). The Spectro-Polarimetric High-contrast Exoplanet REsearch (SPHERE; Beuzit et al. 2008), is an extreme adaptive optics system (Fusco et al. 2014) installed on the VLT in Paranal (Chile). We used SPHERE in its IRDIFS-EXT mode which consists of two instruments working simultaneously, a dual-band imager and spectrograph (IRDIS; Dohlen et al. 2008), and an integral-field spectrograph (IFS; Claudi et al. 2008). We used IRDIS in the dual-band imaging mode (DBI; Vigan et al. 2010) in $\mathrm{K} 12\left(\lambda_{K 1}^{\mathrm{c}}=2.103 \mu \mathrm{m}, F W H M_{K 1}=0.102 \mu \mathrm{m}\right.$; $\left.\lambda_{K 2}^{\mathrm{c}}=2.255 \mu \mathrm{m}, F W H M_{K 2}=0.109 \mu \mathrm{m}\right)$, and IFS in $Y H$ band $(0.95-1.65 \mu \mathrm{m}$, average spectral resolution per FWHM, $R=29$ ). As HD 4747 has a declination very close to the Paranal latitude, the star passes almost at the zenith. This results in a small field rotation in our observation $\left(7.3^{\circ}\right)$, but thanks to the moderate contrast of the companion in the infrared $\left(\Delta K_{1}=9.11 \pm 0.15\right)$, we managed to extract the spectrophotometry and astrometry from our data with a very high accuracy (see Sect. 6).

The observations were done using the standard SHINE strategy. The data sequences consist of the science coronagraph observations in IRDIFS-EXT mode with a detection integration 
time (DIT) of 32s, followed by a star centering dataset with same DITs and with the satellite spots, induced by the deformable mirror, activated in order to recover the position of the star behind the coronagraph (Langlois et al. 2013). Then a flux calibration dataset was produced with DIT $=2 \mathrm{~s}$, with the star offset from the coronagraph, and a neutral density filter (ND_2.0) in order to prevent saturation. A set of sky frames were also taken just after the sequence. The rest of the calibrations (flats, darks, and spectral calibrations) were done at the end of the night with the instrument internal calibration hardware. The astrometric calibration (true north and pixel scale) was done on sky with the SPHERE GTO standard procedure (Maire et al. 2016b). The 2017 epoch was done in the same way as the 2016, but with a shorter coronagraphic sequence as it was mainly aiming to better constrain the orbit.

\subsection{Archival NACO observation}

An archival NACO dataset from 7 September 2008 was also reprocessed to search for and possibly retrieve the separation and position angle of the companion. It was taken in the SDI+4 mode (Maire et al. 2014), which combines the SDI mode of NACO with a four quadrant phase mask coronagraph (Boccaletti et al. 2004). The data was taken in the "double roll subtraction" procedure $^{6}$ with $5^{\circ}$ of rotation offsets every 5 images in order to subtract the speckles linked to pupil aberrations. We obtained a field rotation of $95^{\circ}$, using a single frame DIT of $30 \mathrm{~s}$ for a total observing time of $3 \mathrm{~h}$ and $20 \mathrm{~min}$.

\section{Direct imaging data reduction, astrometry, and spectrophotometric extraction}

The data reductions were performed with three different pipelines, namely the GRAPHIC pipeline (Hagelberg et al. 2016), the LAM-ADI pipeline (Vigan et al. 2015, 2016), and a reduction from the SPHERE Data Center (DC) using the SPHERE Data Reduction and Handling (DRH) automated pipeline (Pavlov et al. 2008) for the standard cosmetic and the SpeCal pipeline for the post-processing (Galicher et al., in prep.). Different types of analysis were also conducted with these pipelines to compare and take full advantage of each of them and to extract the astrometry and spectra of HD 4747B with the best accuracy. We built the SED of the host star (Fig. 3) based on a BT-NextGen model (Allard et al. 2012), and using VOSA (Bayo et al. 2008) to extract the photometry of the primary in the different band filters and find the best fit. All three pipelines gave results similar to the astrometric and photometric standard deviations, respectively 66 and 50\%, smaller than the error bars given hereafter.

\subsection{IRDIS data reduction}

We processed the IRDIS data with several algorithms, angular differential imaging (ADI), spectral differential imaging (SDI), and radial-profile subtraction (Fig. 4). With the small separation ( $\rho \simeq 600$ mas) and field rotation (Sect. 3.2), the ADI and SDI self-subtraction are substantial. However, the companion has a small contrast with respect to its host star in K12 ( $\sim 9 \mathrm{mag})$ and is visible in the raw frames. We therefore decided to use a simple median combination of the frames with a background fit (Fig. 4a) for the photometry extraction in $K_{1}$ and $K_{2}$ and obtained magnitude contrasts of $\Delta K_{1}=9.11 \pm 0.15$ and $\Delta K_{2}=9.24 \pm 0.15$. The

6 See NACO manual for Period 81 and 82: https://www.eso. org/sci/facilities/paranal/instruments/naco/doc/

VLT-MAN-ESO-14200-2761_v81-2.pdf

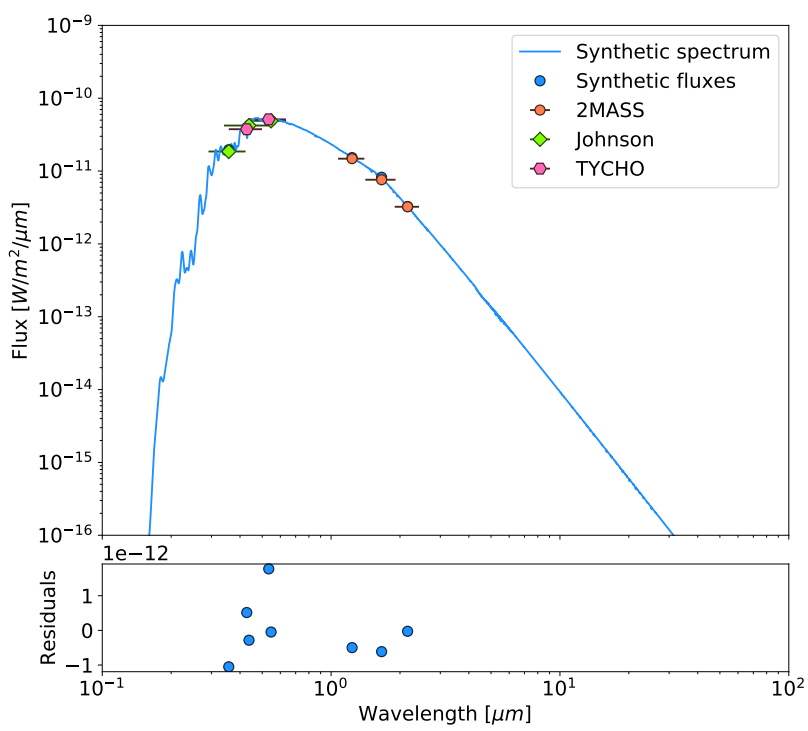

Fig. 3. BT-NextGen synthetic spectrum of HD 4747, scaled to match SED of optical and mid-infrared photometry.

Table 3. Measured astrometry and contrast of HD 4747B from SPHERE $\left(K_{1}\right.$ and $\left.K_{2}\right)$ and NACO (H, SDI mode) data.

\begin{tabular}{ccccc}
\hline \hline Filter & Date (UT) & $\rho$ (mas) & PA (deg) & Contr. (mag) \\
\hline$K_{1}$ & 12.12 .2016 & $594.4 \pm 5.1$ & $187.2 \pm 0.3$ & $9.11 \pm 0.15$ \\
$K_{2}$ & 12.12 .2016 & $595.0 \pm 5.1$ & $187.6 \pm 0.3$ & $9.24 \pm 0.15$ \\
$K_{1}$ & 28.09 .2017 & $581.2 \pm 5.8$ & $190.6 \pm 0.5$ & - \\
$K_{2}$ & 28.09 .2017 & $580.8 \pm 6.3$ & $190.6 \pm 0.7$ & - \\
$H$ & 07.09 .2008 & $608 \pm 11$ & $156.4 \pm 1.3$ & - \\
\hline
\end{tabular}

error bars take into account the error on the PSF fit and the variations in the PSF and speckle noise through the observational sequence. We derived them from the SED of the primary its flux in each filter, and finally extracted the flux of HD 4747B (Fig. 6).

The astrometric extraction was done using a simple radialprofile subtraction (Fig. 4b) as it allowed us to increase the $\mathrm{S} / \mathrm{N}$ and it does not strongly affect the shape of the companion point spread function (PSF). The derived separations and position angles are listed in Table 3. The error bars given in the table include astrometric calibration errors that were quadratically added to the results of the adjustment.

\subsection{IFS data reduction and spectral extraction}

We used a TLOCI-ADI reduction to extract the IFS photometry (Marois et al. 2014, specal implementation used), which was optimized for the best contrast in each wavelength with the least possible impact on the spectrum of the companion. The contrast in each wavelength channel was computed by injecting fake companions before the ADI processing. This allowed us to account for the self-subtraction effect from the ADI reduction. The apparent spectrum of the companion was then extracted with the same procedure as for the IRDIS data by computing the primary flux in each channel given its SED. The extracted spectrum is shown in Fig. 6.

\subsection{NACO data reduction and astrometric extraction}

We reduced the NACO data following the standard data processing, bad-pixel cleaning, subtraction of sky, and division by flats. The SDI subimages were extracted and recentered by adjusting 


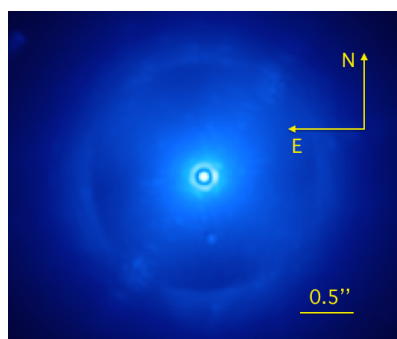

(a)

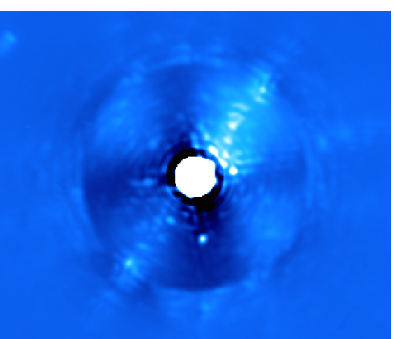

(b)

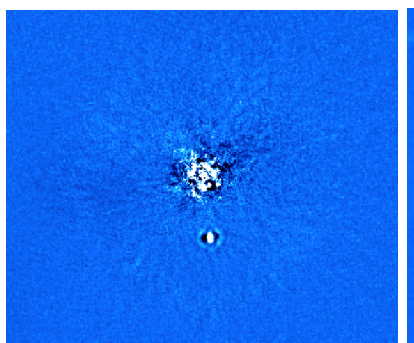

(c)

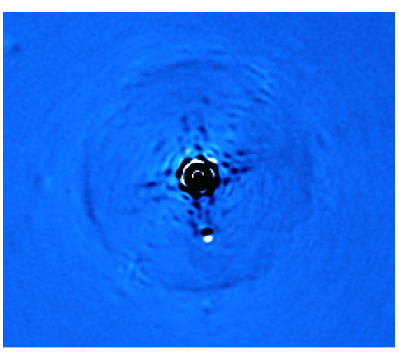

(d)

Fig. 4. Four IRDIS reductions of HD 4747. Panel $a$ : IRDIS reduction using median combine only. Panel $b$ : IRDIS reduction using radial-profile subtraction. Panel $c$ : IRDIS reduction using ADI (TLOCI). Panel $d$ : IRDIS reduction using SDI and simple derotation. The companion is observed in each reduction. The north and east directions and the scale are indicated in image $(a)$.

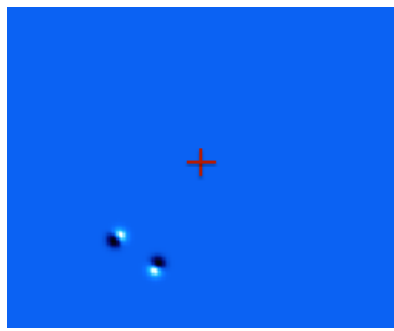

(a)

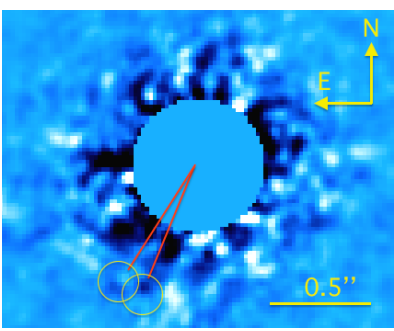

(b)

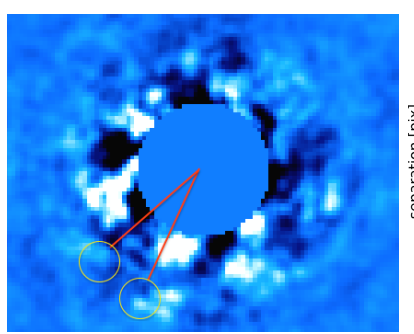

(c)

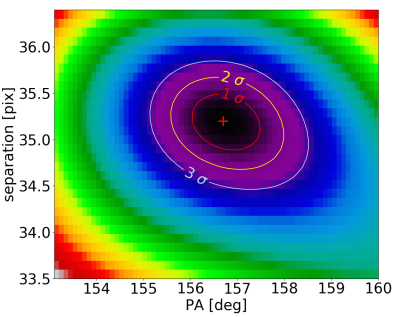

(d)

Fig. 5. NACO final images after SDI and double roll subtraction applied for different angles $\left(5^{\circ}, 10^{\circ}\right.$, and $\left.25^{\circ}\right)$, and final $\chi^{2}$ map of separation vs. position angle. Panel $a: 25^{\circ}$ double roll subtraction angle model. Panel $b$ : $10^{\circ}$ double roll subtraction angle. Panel $c$ : $25^{\circ}$ double roll subtraction angle. Panel $d$ : median $\chi^{2}$ map over double roll subtraction angles. The cross shows the minimum $\chi^{2}$ and thus the position of the companion.

a simple Gaussian profile to the wings of the primary star. We performed a frame selection based on the integrated flux of each image. Images with a flux higher than the median value were rejected, which allowed us to remove frames taken with lower seeing conditions and/or AO performance. The shorter wavelength images were resized by a factor $\lambda 1 / \lambda 2$ and centered by the cross-correlation to the longer wavelength images. For each time step, the images taken at different wavelengths were subtracted. Images with the same derotator angle were co-added using their median value.

Finally, different sets of double roll subtractions were performed to remove residual pupils aberrations and to amplify the companion signature. For instance, we subtracted every image pair that has a pupil rotation offset of $10^{\circ}$, i.e., $\operatorname{Im}\left(10^{\circ}\right)-\operatorname{Im}\left(0^{\circ}\right)$, $\operatorname{Im}\left(15^{\circ}\right)-\operatorname{Im}\left(5^{\circ}\right), \ldots, \operatorname{Im}\left(95^{\circ}\right)-\operatorname{Im}\left(85^{\circ}\right)$, and median combined them. We did the same on image pairs with $5^{\circ}, 15^{\circ}, 20^{\circ}$, and $25^{\circ}$ pupil rotation offsets. This data analysis technique affects the companion signature in the final image, which is composed of four duplicated PSF as shown in Fig. 5a. The SDI part of the algorithm results in the radial positive/negative part of the companion signature, while the double roll subtraction duplicates the SDI pattern with the chosen rotation offset. We found that the $10^{\circ}$ pupil rotation offset performs the best in terms of noise reduction and signal amplification (see Fig. 5b), which allowed us to detect HD 4747B in the archived NACO data. We retrieved the companion astrometry and the corresponding confidence intervals by modeling the expected companion pattern and computing a $\chi^{2}$ map for different companion separations, position angles, and flux ratios. A clear minimum in the $\chi^{2}$ map is seen at the expected position of the companion as illustrated in Fig. 5d. Each individual full $\chi^{2}$ maps for the different double roll subtraction angles are shown in Fig. A.1, while Fig. A.1f and Fig. 5d show the median (see Appendix A for more details of this analysis).

Deriving reliable confidence intervals for these archived data is also a challenging task. We computed the noise of the image

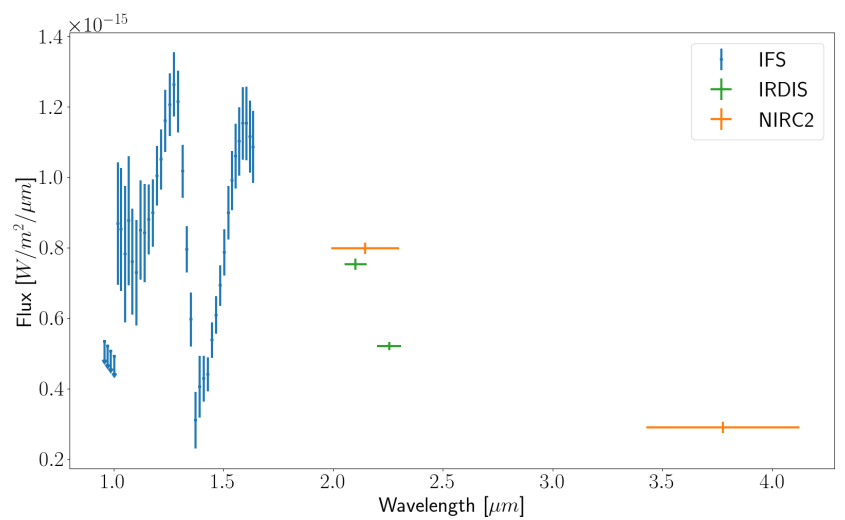

Fig. 6. Extracted spectrophotometry of HD 4747B. The blue points are SPHERE IFS data, the green and yellow points are respectively IRDIS SPHERE and NIRC2 photometry. The NIRC2 data are taken from Crepp et al. (2016). The horizontal errorbars represent the width of the IRDIS and NIRC2 filters.

for each separation using the standard deviation in annulus of 1 pixel width. This noise map was used to compute the $\chi^{2}$ map and the 1,2, and $3 \sigma$ confidence intervals (see Fig. 5d). As the center of the star is difficult to determine behind the coronagraph, we decided to add quadratically a systematic error of 4 mas on the separation measurement, which corresponds to a quarter of a pixel. As no astrometric field was observed during this run with NACO, and no calibration could be found for the SDI+4 mode's pixel scale, we took the NACO manual value of 17.32 mas $^{-1}$. We quadratically added an error of $0.05 \mathrm{smas}_{\mathrm{pixel}}{ }^{-1}$ to the budget value, which corresponds to an error of 1.8 mas at the separation of the companion. For true north, we used the calibration from Ehrenreich et al. (2010), taken on 20 August 2008, 18 days before our dataset. The true north of NACO was at a $0^{\circ} \pm 0.2^{\circ}$ angle. We quadratically added an uncertainty of $0.5^{\circ}$ as the detector could have moved slightly 
between the runs. An angular separation of $\rho=608 \pm 11$ mas and a position angle of $\mathrm{PA}=156.4^{\circ} \pm 1.3^{\circ}$ were retrieved.

We decided not to use the photometry of this dataset. The detection is at the noise limit, and the flux is strongly polluted by the self-subtraction of both SDI and the double roll subtraction. Moreover, the filters are redundant with the SPHERE IFS channels and the PSF calibration flux was not correctly done.

\section{Orbital analysis and dynamical mass estimation}

We performed a Markov chain Monte Carlo (MCMC) analysis combining all radial velocity data available (see Sect. 3.1 and Fig. 7) with our direct imaging SPHERE epochs (see Sect. 4 and Fig. 8), the epochs from Crepp et al. (2016) and the NACO SDI+4 archival data point (see Sect. 4.3). This epoch allowed us to constrain strongly the orbital parameters thanks to the much longer time baseline. The MCMC simulation was performed by using emcee (Foreman-Mackey et al. 2013), a python implementation of the affine-invariant ensemble sampler for MCMC proposed by Goodman \& Weare (2010). The data were modeled with a Keplerian and four RV offsets (one for HIRES, and threee for the different versions of CORALIE: C98, C07, C14). The noise in the radial velocity data was modeled with a nuisance parameter for each instrument. As we have direct imaging and radial velocity measurements, the parallax and mass of the primary are parameters that can be constrained with the fitting. We introduce these two parameters with Gaussian priors taken from Table 2. More details of the MCMC orbital analysis are presented in Appendix B. The results of the orbital fit are presented in Table 4, and Figs. B.1 and B.2.

Compared to the orbital solutions found by Crepp et al. (2016), we find significant differences in the results of our MCMC analysis. The period $P=33.08 \pm 0.70 \mathrm{yr}$ is in good agreement with Sahlmann et al. (2011b), but disagrees with the Crepp et al. (2016) value at $6 \sigma$. The high eccentricity predicted in Sahlmann et al. (2011b) and confirmed by Crepp et al. (2016) is verified, $e=0.7320 \pm 0.0023$, even if our value is slightly lower. The inclination of $46.3^{\circ} \pm 1.1^{\circ}$ derived leads to a semi-major axis of $a=10.01 \pm 0.21$ au and a mass estimation for HD 4747B of $m_{\mathrm{B}}=70.2 \pm 1.6 M_{\odot}$. This inclination is smaller by $3 \sigma$ than the value from Crepp et al. (2016). This explains the difference in the mass measurement. The discrepancy between our orbital parameters and those of Crepp et al. (2016) likely arises from the Keck $2015 L^{\prime}$ band measurement. This datapoint is off by more than $1 \sigma$, and with more epochs our fit is less sensitive to individual outliers.

\section{Spectrophotometric analysis}

\subsection{Color-magnitude diagram}

From the IRDIS $K_{1}$ and $K_{2}$ observations the color-magnitude diagram of HD 4747B shows a late L spectral type (Fig. 9). This is in good agreement with the prediction of Crepp et al. (2016), which used the broadband $K s$ and $L^{\prime}$ filters from the NIRC2 camera at the Keck Telescope. HD 4747B falls close to the HR8799 $\mathrm{c}, \mathrm{d}$, and e planets in the diagram and at the $\mathrm{L}-\mathrm{T}$ transition.

\subsection{Known standards comparison}

To further constrain the spectral type of HD 4747B, a comparison of standard near-IR spectra of L1 to T8 known objects from the SpeX Prism Library (Burgasser 2014) was done. We made use of the splat python package (Burgasser et al. 2016). Each standard spectrum is first normalized in flux and then calibrated
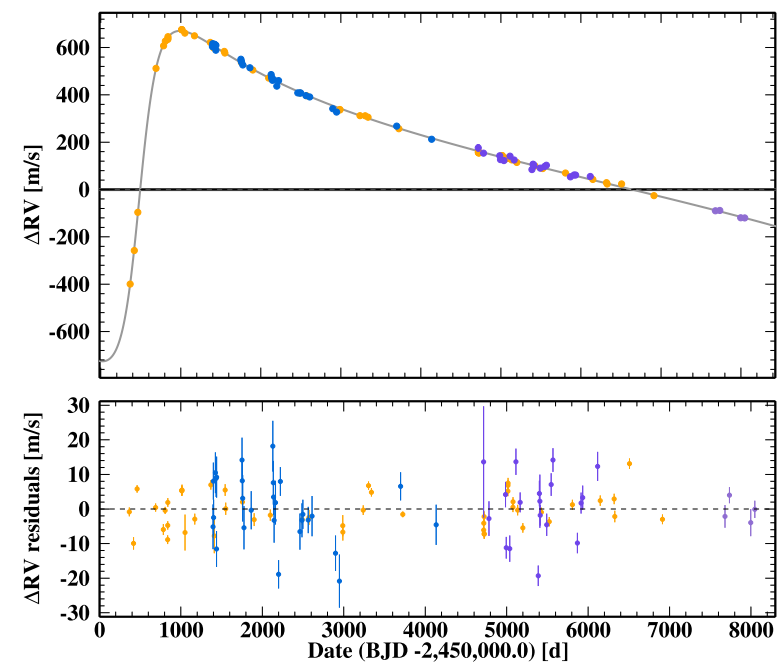

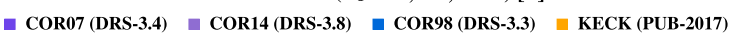

Fig. 7. Radial velocity measurements for HD 4747 taken with the Keck-HIRES and CORALIE spectrographs. The different versions of CORALIE are indicated with different colors. The best-fit model from the combined MCMC analysis with direct imaging is marked with the gray line.

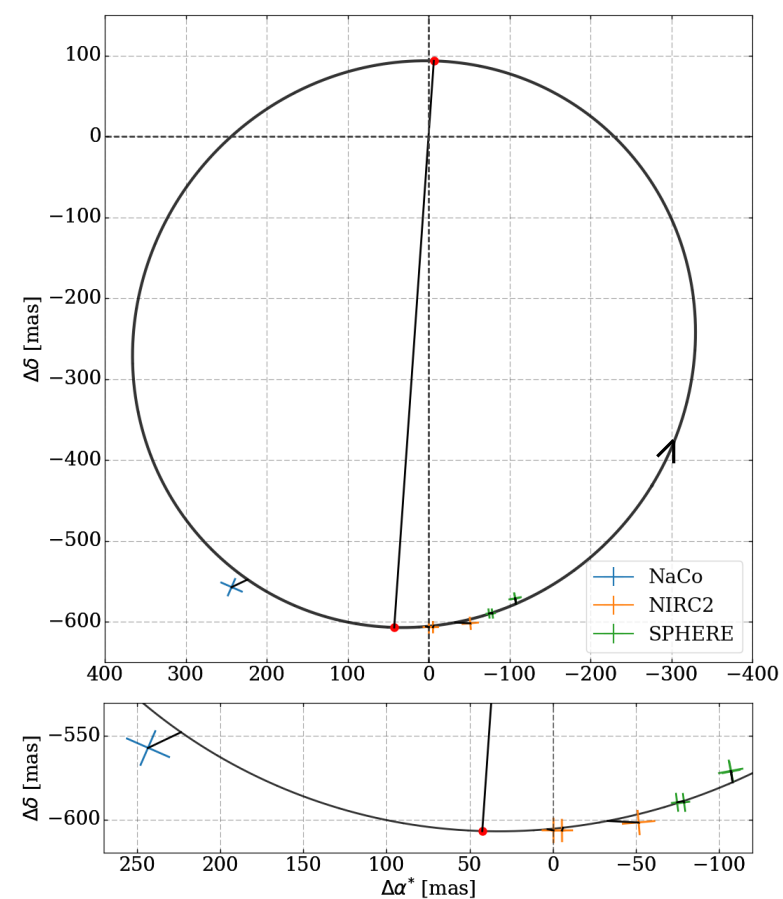

Fig. 8. Relative orbit of the HD 4747AB system. The black curve corresponds to the maximum likelihood of the combined RV and direct imaging MCMC analysis. The $\mathrm{NaCo}$ data point is shown in blue, the NIRC2 $K s$ and $L^{\prime}$ observations from Crepp et al. (2016) in yellow, and the SPHERE ones in green. The arrow shows the orbit rotational direction and the red dots show apastron and periastron.

to match the extracted data of HD 4747B. The fitting of each standard spectrum $\left(F_{\mathrm{k}}\right)$ on the one of HD 4747B $(f)$ is done by comparing the goodness of fit

$\chi^{2}=\sum_{i=1}^{n}\left(\frac{f_{\mathrm{i}}-C_{\mathrm{k}} F_{\mathrm{k}, \mathrm{i}}}{\sigma_{\mathrm{i}}}\right)^{2}$,

where $C_{\mathrm{k}}$ is the flux scaling factor. Each standard spectrum was binned to the appropriate spectral resolution of the IFS measurements with a Gaussian convolution. We decided to use a FWHM 
Table 4. Orbital parameters from the maximum likelihood.

\begin{tabular}{cc}
\hline \hline Parameters & Values $(1 \sigma)$ \\
\hline$P[\mathrm{yr}]$ & $33.08 \pm 0.70$ \\
$K[\mathrm{~m} / \mathrm{s}]$ & $698.0 \pm 10.4$ \\
ecc & $0.7320 \pm 0.0023$ \\
$\omega[\mathrm{deg}]$ & $-93.10 \pm 0.47$ \\
$T_{0}[\mathrm{bjd}]$ & $50473.9 \pm 5.2$ \\
$\Omega[\mathrm{deg}]$ & $89.9 \pm 1.4$ \\
$i[\mathrm{deg}]$ & $46.3 \pm 1.1$ \\
$m \sin (i)\left[M_{\mathrm{Jup}}\right]$ & $50.7 \pm 1.8$ \\
\hline$m_{1}+m_{2}\left[M_{\odot}\right]$ & $0.918 \pm 0.037$ \\
$m_{2}\left[M_{\mathrm{Jup}}\right]$ & $70.2 \pm 1.6$ \\
$a[\mathrm{au}]$ & $10.01 \pm 0.21$ \\
\hline
\end{tabular}

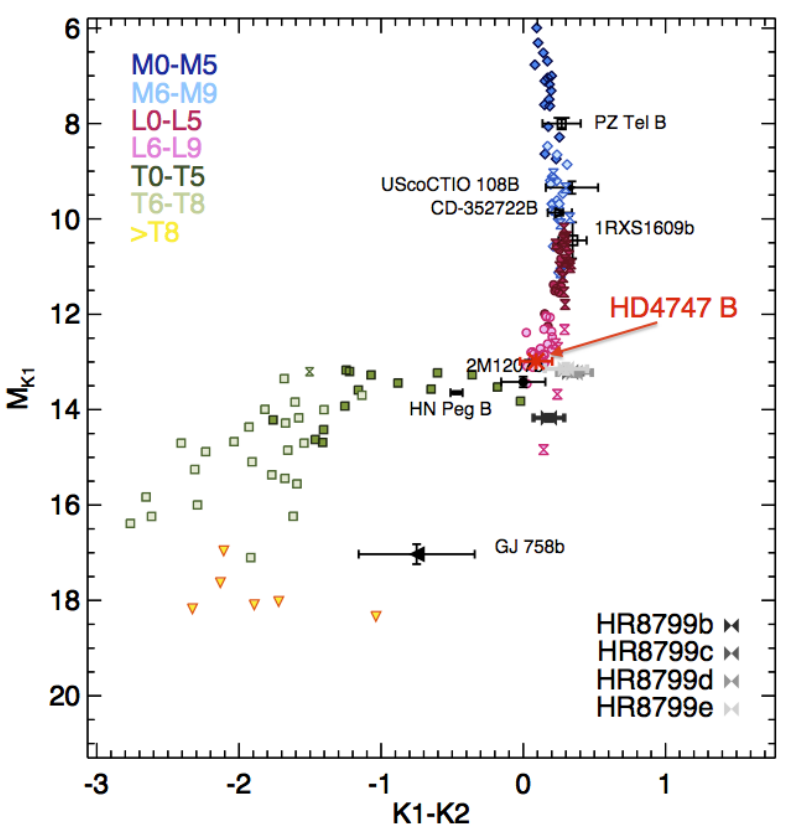

Fig. 9. Color-magnitude diagram of HD 4747B from the IRDIS $K_{1}$ and $K_{2}$ observations. Other known objects are shown for comparison.

of 1.5 times the separation between each wavelength in order to take into account the correlation between the IFS channels. The IRDIS fluxes were estimated by using the transmission curves of the $K_{1}$ and $K_{2}$ filters. The $L^{\prime}$ NIRC2 observation was not used as the spectra from this library stop in the $K$ band. Figure 10 shows the fitting results for the L8, L9, and T0 standards. The best fit corresponds to the L9 dwarf DENIS-P J0255-4700 (Burgasser et al. 2006) and matches the IFS and IRDIS data well. This is in good agreement with the color-magnitude diagram (Fig. 9). Crepp et al. (2016) also derived a late L type, but a higher effective temperature of $T_{\text {eff }}=1700 \pm 100 \mathrm{~K}$. This led them to a model dependent mass that is higher than in this study.

\subsection{Atmospheric forward modeling: Exo-REM}

We characterized the observations with the forward model ExoREM (Baudino et al. 2015, 2017) using grids of synthetic models generated with a $T_{\text {eff }}$ between 400 and $1800 \mathrm{~K}$ (with a step of $50 \mathrm{~K})$, a $\log (g[\mathrm{cgs}])$ between 2.5 and 5.5 (with a step of 0.5 ), a diversity of clouds (without cloud, or with $\tau_{\text {ref }}=0.5$ or 1 ; see Baudino et al. 2015), a metallicity $z=-0.2,0,0.5,1.0$ and taking account of the equilibrium chemistry or non-equilibrium chemistry (with a $k_{\mathrm{zz}}=10^{8} \mathrm{~cm}^{2} \mathrm{~s}^{-1}$, see Baudino et al. 2017 for the

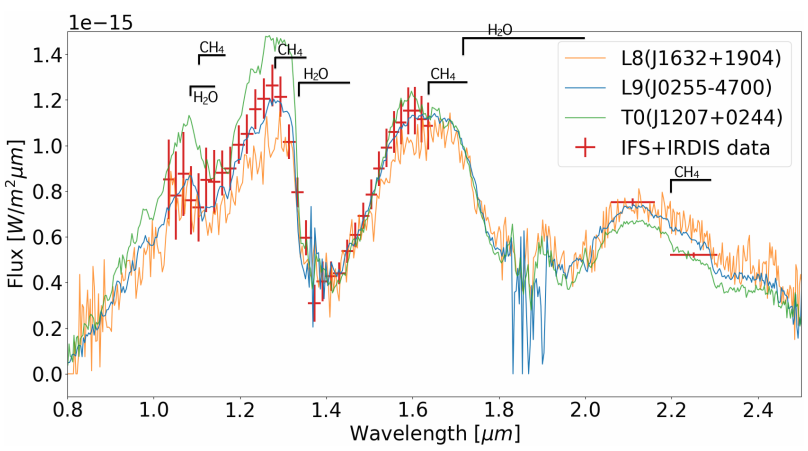

Fig. 10. Adjustments of standard known objects showing L8 (J16322911+1904407; Burgasser 2007a), L9 (J02550357-4 700 509; Burgasser et al. 2006), and T1 (J12074717+0244249; Looper et al. 2007). The best fit is obtained by the L9 object. The molecular absorption bands of $\mathrm{CH}_{4}$ and $\mathrm{H}_{2} \mathrm{O}$ are indicated.

Table 5. Results of the forward modeling Exo-REM at $5 \sigma$ detection for HD 4747B.

\begin{tabular}{ll}
\hline \hline Parameter & Values \\
\hline$T_{\text {eff }}(\mathrm{K})$ & $1300 \pm 100$ \\
$\log (g)($ dex $)$ & $4 \pm 0.5$ \\
Cloud condition & Cloudy \\
Chemistry & No conclusion \\
Radius $\left(R_{\text {Jup }}\right)$ & $0.91 \pm 0.16$ \\
Metallicity (solar) & $0.63-1$ \\
\hline
\end{tabular}

non-equilibrium chemistry formalism). The parameters of the best model are summarized in Table 5 and the best-fit spectra are shown in Fig. B.3. The maximum mass at $1 \sigma$ computed from the gravity and radius derived with Exo-REM is only $14.6 M_{\text {Jup }}$, which is far below the dynamical mass measurement. The difference between the results with Exo-REM and the dynamical mass can be explained by the difficulty generating the more extreme surface gravity with this model (built for giant planets, i.e., lowgravity objects). HD 4747B is indeed the most massive brown dwarf studied with Exo-REM and is at the gravity limit available with this model.

\subsection{Atmospheric retrieval modeling: HELIOS- $R$}

For this analysis we used the atmospheric retrieval code HELIOS-R developed by Lavie et al. (2017). Atmospheric retrieval is a technique borrowed from the Earth remote sensing community. Some pieces of the atmospheric physical model are parametrized (i.e., Temperature-pressure profile, clouds, etc.). It sacrifices self-consistency in order to speed up computational time, which in return allows a more robust parameter space exploration and a better characterization of the uncertainties on the model parameters.

HELIOS-R allows a direct comparison of different 1D emission forward model using the Nested Sampling algorithm (Skilling 2006). The model parameters and their priors are presented in Table 6. As in Lavie et al. (2017), we assume independent Gaussian errors, so the likelihood takes the form of Eq. (2)

$\mathcal{L}\left(D \mid M_{i}, \theta\right)=\prod_{k=1}^{N} \frac{1}{\sigma_{k} \sqrt{2 \pi}} \exp \left(-\frac{\left[D_{k, \text { obs }}-D_{k, \text { model }}\right]^{2}}{2 \sigma_{k}^{2}}\right)$,

where $D_{k \text { obs }}$ is the kth observational data point, $D_{k \text {,model }}(\theta)$ the model prediction for this data point given the parameters $\theta$, 
Table 6. Parameters and priors used in the retrieval analysis.

\begin{tabular}{llll}
\hline \hline Parameter & Symbol & Prior used & Value \\
\hline Radius & $R$ & Gaussian & $R_{\text {comp }}=1.0 \pm 0.1 R_{\text {Jup }}$ \\
Planet mass & $M_{\text {comp }}$ & Gaussian & $70.2 \pm 1.6 M_{\text {Jup }}$ \\
Molecule abundances or elemental abundances & $X_{i}, f_{i}$ & Log-uniform & $10^{-15}-10^{-1}$ \\
Longwave/infrared opacity (TP profile) & $\kappa_{0}$ & Log-uniform & $\log \kappa_{0}=10^{-15}-10(\mathrm{mks})$ \\
Internal/interior temperature (TP profile) & $T_{\text {int }}$ & Uniform & $100-2300 \mathrm{~K}$ \\
Extinction coefficient & $Q_{0}$ & Uniform & 1 to 100 \\
Cloud particle size & $r_{\mathrm{c}}$ & Log-uniform & $10^{-7}-10^{-3} \mathrm{~m}$ \\
Cloud particle abundance & $f_{\text {cloud }}$ & Log-uniform & $10^{-30}-10^{-4}$ \\
Distance & $d$ & Gaussian & $19.25 \pm 0.58 \mathrm{pc}$ \\
\hline
\end{tabular}

$\sigma_{k}$ the uncertainty of the kth observational data point, and $\mathrm{N}$ the total number of data points. This approach does not account for covariances in the IFS data.

The models assume a hydrogen/helium dominated atmosphere and include the four main absorbers in the infrared: carbon monoxide $(\mathrm{CO})$, carbon dioxide $\left(\mathrm{CO}_{2}\right)$, water $\left(\mathrm{H}_{2} \mathrm{O}\right)$, and methane $\left(\mathrm{CH}_{4}\right)$. Two sets of assumptions can be made regarding the atmosphere chemistry: equilibrium chemistry, where the two parameters are the carbon $\left(f_{\mathrm{c}}\right)$ and oxygen $\left(f_{\mathrm{o}}\right)$ abundances, and unconstrained chemistry, where the parameters are the four molecule abundances assumed to be constant throughout the vertical 1D atmosphere. Clouds are modeled using the threeparameter model first introduced in Lee et al. (2013; see Lavie et al. 2017 for more insight on HELIOS-R).

\subsubsection{Companion mass, gravity, and radius priors}

As discussed in Lavie et al. (2017), retrieving the surface gravity and radius of directly imaged objects is challenging. With the current number of atmospheric data (spectrum and photometry) and their uncertainties, it is impossible with an atmospheric retrieval technique to constrain the surface gravity at the same level of precision as with the radial velocity data. In the case of HD 4747B, velocity data are available. This valuable information needs to be taken into account in the atmospheric Bayesian analysis. We have updated HELIOS-R in order to take the companion mass as a parameter of the model. The mass, surface gravity, and radius are linked by the following equation:

$M_{\text {comp }}=g * R_{\text {comp }}^{2} / G$

The companion mass constrained by the radial velocity can now be enforced in our prior in a straightforward manner. There is no direct measurement of the radius on this object as it does not transit. Consequently, our prior should reflect our current state of knowledge on the radius of brown dwarfs. The evolutionary track (e.g., Baraffe et al. 2003) gives a radius of $0.9 R_{\text {Jup }}$ for HD 4747B. However, there is not a unique mass-radius relationship for a given brown dwarf depending on what one assumes in the models (Burrows et al. 2011). Konopacky et al. (2010) also showed that traditional evolutionary tracks are missing physics or chemistry. Observations of transiting brown dwarfs show that those objects have radii from $\sim 0.8$ to $1.2 R_{\text {Jup }}$. We therefore set our prior as a Gaussian prior of $R=1.0 \pm 0.1 R_{\mathrm{Jup}}{ }^{7}$.

\subsubsection{Results and discussion}

The main output of the nested sampling algorithm is the Bayesian evidence, which allows the comparison of the models

\footnotetext{
7 This Gaussian prior takes into account the 0.8-1.2 $R_{\text {Jup }}$ distribution at $2 \sigma$.
}

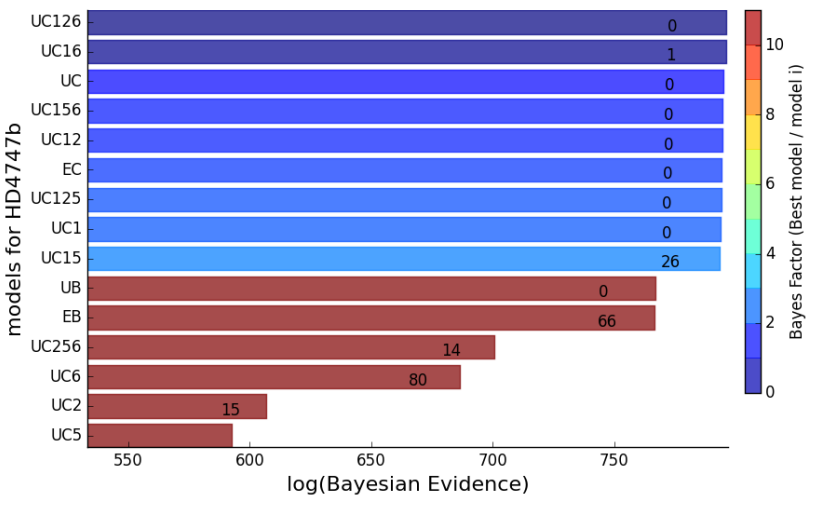

Fig. 11. Bayes factors from a suite of models for HD 4747B. Four models were considered: equilibrium chemistry without clouds (EB) or with clouds (EC), and unconstrained chemistry without clouds (UB) or with clouds (UC). The numbers associated with each model's name indicate the molecules included $\left(1: \mathrm{H}_{2} \mathrm{O}, 2: \mathrm{CO}_{2}, 5: \mathrm{CO}\right.$, and 6: $\left.\mathrm{CH}_{4}\right)$; if there are no numbers then all four molecules are included. The Bayesian evidence clearly favors models with clouds and the most favored model is unconstrained chemistry including $\mathrm{H}_{2} \mathrm{O}, \mathrm{CO}_{2}$, and $\mathrm{CH}_{4}$. The number associated with each histogram is the logarithm of the Bayes factor between the model in question and its neighbor below. The color bar shows the logarithm of the Bayes factor between the model in question and the best model, which is the model placed at the top.

through the computation of the Bayes factor $(\mathcal{B})$. We considered four different models: equilibrium chemistry without clouds (EB) or with clouds (EC), and unconstrained chemistry without clouds (UB) or with clouds (UC). For the models with unconstrained chemistry, we also considered all the different combinations of molecules in order to evaluate the significance of each molecule detection. Figure 11 shows the Bayesian evidence for most of the models considered. Models with clouds are strongly preferred over the models without clouds, which indicates that this object is probably cloudy. The unconstrained chemistry model with clouds is the preferred model. The Bayes factor between this model (including all the molecules) and the models excluding some molecules indicate that $\mathrm{CO}_{2}$ and $\mathrm{CH}_{4}$ are only weakly detected $(\ln \mathcal{B}<2.5)$, while $\mathrm{H}_{2} \mathrm{O}$ is strongly detected $(\ln \mathcal{B}>5)$. We have a low sensitivity to $\mathrm{CO}$; including it or not in the model does not change the constraints on the other parameters and does not improve the fit to the data (see also Trotta 2008 for a correspondence between the Bayes factor (B) and the significance in terms of the number of standard deviations). A summary of the retrieved parameters for the UC model (including all the molecules) is shown in Table 7, the best-fit spectrum and temperature profile are shown in Fig. C. 2 and the posterior distributions of the parameters are shown in Fig. C.3. 
Table 7. Summary of retrieved results.

\begin{tabular}{lc}
\hline \hline Property & Value \\
\hline$X_{\mathrm{H}_{2} \mathrm{O}}$ & $-3.57_{-0.07}^{+0.07}$ \\
$X_{\mathrm{CO}_{2}}$ & $-6.13_{-5.11}^{+1.92}$ \\
$X_{\mathrm{CO}}$ & $-9.65_{-3.08}^{+3.28}$ \\
$X_{\mathrm{CH}_{4}}$ & $-4.62_{-0.43}^{+0.25}$ \\
$Q_{0}$ & $0.88_{-0.50}^{+0.58}$ \\
$r_{\mathrm{c}}[\mathrm{m}]$ & $-4.57_{-0.73}^{+0.86}$ \\
$X_{\mathrm{c}}$ & $-21.00_{-1.56}^{+1.32}$ \\
$d[\mathrm{pc}]$ & $19.56_{-0.41}^{+0.35}$ \\
$M_{\mathrm{p}}\left[\mathrm{M}_{\mathrm{Jup}}\right]$ & $70.09_{-1.21}^{+1.25}$ \\
$R_{\mathrm{p}}\left[\mathrm{R}_{\mathrm{Jup}}\right]$ & $0.85_{-0.03}^{+0.03}$ \\
\hline$\mu$ & $2.20_{-0.00}^{+0.00}$ \\
$\mathrm{C} / \mathrm{O}$ & $0.13_{-0.08}^{+0.14}$ \\
$\mathrm{C} / \mathrm{H}$ & $-4.72_{-0.39}^{+0.47}$ \\
$\mathrm{O} / \mathrm{H}$ & $-3.79_{-0.08}^{+0.16}$ \\
$\log g[\mathrm{cgs}]$ & $5.40_{-0.03}^{+0.03}$ \\
\hline &
\end{tabular}

Notes. We have listed the $1 \sigma$ uncertainties, which were computed by locating the 15.87th and 84.13th percentile points on the horizontal axis. In the limit of a symmetric Gaussian function, these would yield its fwhm. The mean molecular weight is given at 1 bar. Values are in $\log _{10}$ (except for $\mathrm{C} / \mathrm{O}, \mathrm{d}, M_{\mathrm{p}}, R_{\mathrm{p}}$, and $\mu$ ) and dimensionless (except when units are shown).

The dimensionless cloud parameter $Q_{0}$ serves as a proxy for the cloud composition. It is not possible to determine the composition as the posterior distribution is too broad and encompasses the refractory species composition (e.g., silicates $-Q_{0} \sim 10$ ) and the volatile species composition (e.g., ammonia, methane, water $\left.-Q_{0} \sim 40-80\right)$. However, the posterior distribution of the cloud particle size indicates that the cloud is composed of big particles, which will act as constant absorbers as a function of the wavelength (Lavie et al. 2017).

The retrieved posterior distribution of the companion mass reflects our prior, which is normal as the radial velocity data (used to build our prior) provide a better constraint on this parameter than the spectrum does. With the retrieved companion radius $\left(0.85 R_{\mathrm{Jup}}\right)$ and mass we are able to compute the surface gravity of the companion using Eq. (3), which indicates a high-gravity object $(\log g=5.40 \mathrm{cgs})$.

We have measured oxygen abundances by exploiting the O I triplet at 7771-7775 Angstroms, and applying 3D NLTE corrections by Amarsi et al. (2015), as done in D'Orazi et al. (2017). We have found a slight overabundance being $[\mathrm{O} / \mathrm{Fe}]=+0.16 \pm$ 0.08 dex. Considering our carbon abundance estimation given in Sect. 2, we obtained $\mathrm{C} / \mathrm{O}=0.417$. Using the retrieved molecular abundances, it is possible to derive the ratios of carbon to oxygen $(\mathrm{C} / \mathrm{O})$, carbon to hydrogen $(\mathrm{C} / \mathrm{H})$, and oxygen to hydrogen $(\mathrm{O} / \mathrm{H})$. The retrieved values are shown in Table 7 . Figure 12 shows a comparison of those values with the HR 8799 system from Lavie et al. (2017; with data from Bonnefoy et al. 2016; Zurlo et al. 2016). These values can be compared to the stellar abundance, which are $10^{-3.82}$ and $10^{-3.44}$ for carbon and oxygen, respectively, derived from the results given in Sect. 2 .
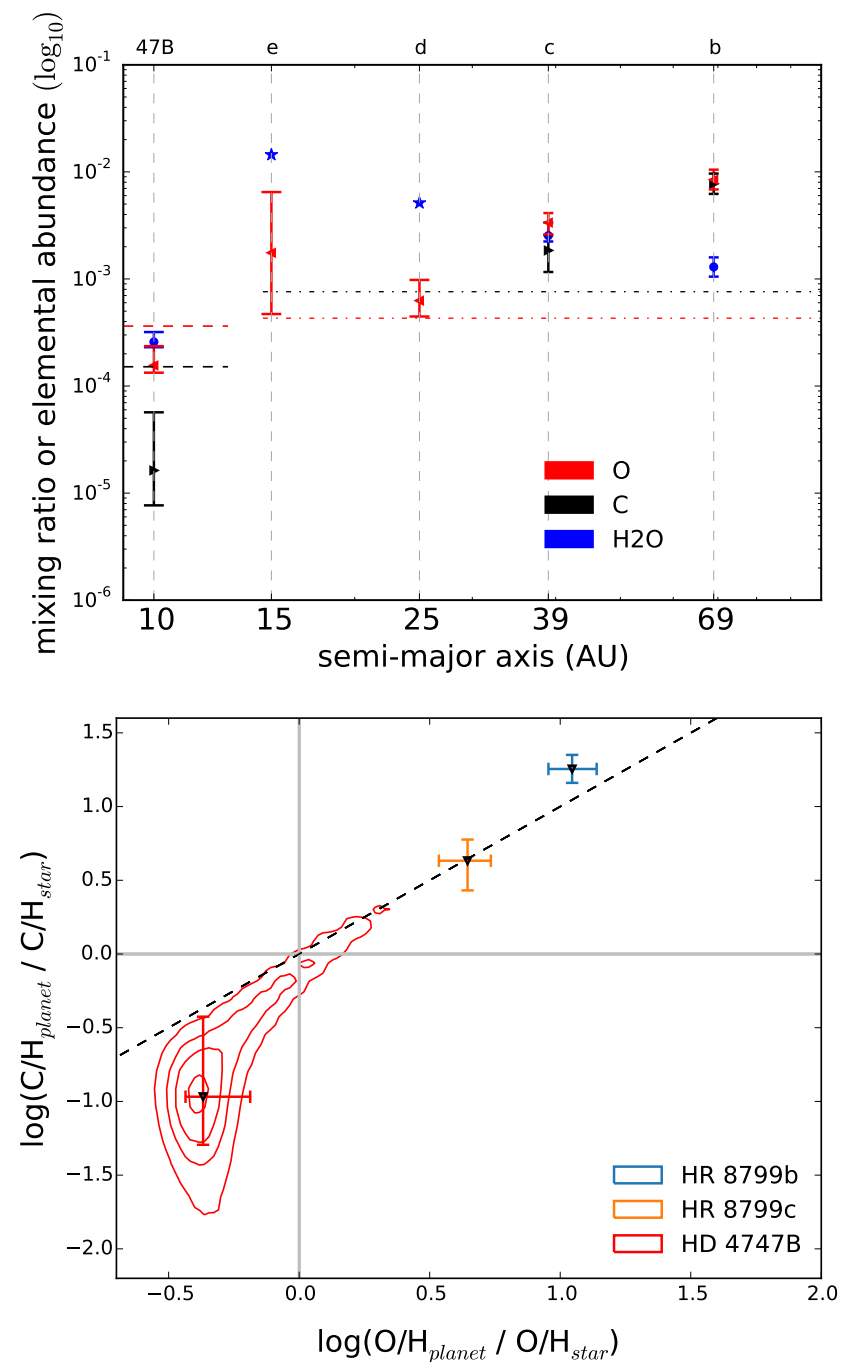

Fig. 12. Results from the atmospheric retrieval analysis. Top panel: the retrieved water mixing ratios and elemental abundances of carbon and oxygen for HD 4747B, and for the four HR 8799 exoplanets as a function of the distance to the host star. For HR 8799d and e, we show the water abundance in chemical equilibrium at 1 bar (blue stars). The carbon abundance retrieved for these two planets is very low and is not shown (see Fig C.4). The carbon and oxygen abundances of the stars are shown with the dashed lines (HD 4747) and dash-dotted lines (HR 8799). Bottom panel: the companion elemental abundances normalized to its stellar values with the dashed black line denoting parity.

The interest in $\mathrm{C} / \mathrm{O}, \mathrm{C} / \mathrm{H}$, and $\mathrm{O} / \mathrm{H}$ lies in the implications for planet formation. Öberg et al. (2011) previously outlined a first-order scenario based on the position of the different snow lines. The core accretion scenario is a multi-step process that will result in a broad-range elemental composition depending on the position of the object during those different steps. The HR 8799 planets are compatible with such a scenario. On the other hand a gravitational instability scenario is a quick one-step process that will form a companion with a similar composition to the host star formed from the same protoplanetary disk. Our retrieved values for HD 4747B are compatible within one sigma to the host star, which indicates that both scenarios are possible. However, the relative low mass of the star and the high mass of the companion indicate that a core accretion scenario would be difficult. It is therefore very likely that this system has formed as a binary system. More observations, especially spectroscopicalobservations in the $K$ band to constrain the carbon abundance 


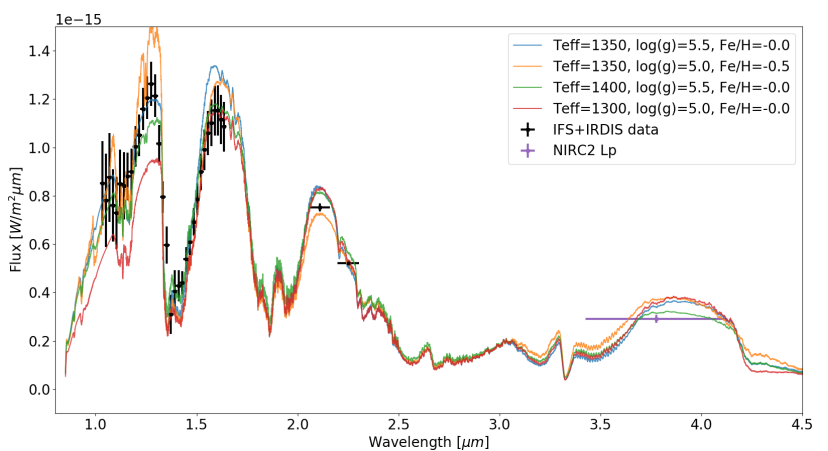

Fig. 13. Four best-fit spectra from the BT-Settl models (Allard et al. 2011) in order from the top (best) to the bottom (less good). The adjustment was done on a grid of $T_{\text {eff }}$ from 1200 to $1700 \mathrm{~K}$, with a $\log (g)$ from 4.0 to 5.5 and a metallicity of $+0.5,0.0$, or -0.5 . The results are consistent with a $T_{\text {eff }}=1300-1400 \mathrm{~K}, \log (g)=5-5.5$, and $[\mathrm{Fe} / \mathrm{H}]=0.0 /-0.5$.

(Lavie et al. 2017), are requested to confirm this scenario through the atmospheric retrieval analysis.

\subsection{BT-Settl model comparison}

To compare the Exo-REM forward modeling and HELIOS-R retrieval with evolutionary models, we used the BT-Settl atmospheric models (Allard et al. 2011) combined with the BHAC15 evolutionary tracks (Baraffe et al. 2015). This model is well suited to analyzing objects that are at the $\mathrm{L}-\mathrm{T}$ transition, such as HD 4747B. We compared our extracted spectrum to model spectra with $T_{\text {eff }}$ from 1200 to $1700 \mathrm{~K}$ (with steps of $50 \mathrm{~K}$ ), with a $\log (\mathrm{g})$ from 4.0 to 5.5 (steps of 0.5 ), and a metallicity of $+0.5,0.0$, or -0.5 . The four best-fitting spectra (binned by a factor of 1000) are shown in Fig. 13. The results point towards a temperature $T_{\text {eff }}=1300-1400 \mathrm{~K}, \log (g)=5.0-5.5$, and a metallicity between 0.0 and -0.5 . The temperature agrees well with the result from the standard-objects fitting and ExoREM forward modeling. The gravity is in good agreement with the HELIOS-R retrieval code. The metallicity is in good agreement with the metallicity of the host $\operatorname{star}([\mathrm{Fe} / \mathrm{H}]=-0.23 \pm 0.05$, see Table 2), even if a thinner grid would be needed to constrain this parameter correctly.

We observe that the peak at $1.2-1.3 \mu \mathrm{m}$ is not well fitted by the BT-Settl models, despite the good match found with the spectra of standard objects. It could mean that the opacity at this wavelength is not perfectly computed in these models, or that the grid of spectra we used is not dense enough in metallicity or in $\log (g)$.

We can compare our spectrum, the estimated age of the system, and the model-independent dynamical mass measurement with the predictions of evolutionary models. Using the measured effective temperature, which combines the information of the overall spectrum, the BHAC15 models (Baraffe et al. 2015) predict a mass around $65 M_{\text {Jup }}$ (Fig. 14). The BHAC15 evolutionary models clearly tend to overestimate the temperature for a given age and mass in this range of mass. In other words the object seems to cool faster than the models predict. However, the age uncertainty is quite high, and a more accurate value as well as thinner model grids at the H-burning limit would be needed to constrain the models correctly.

\section{Conclusion}

HD 4747B is a useful mass-age-metallicity benchmark object for comparison with brown dwarf atmospheric and

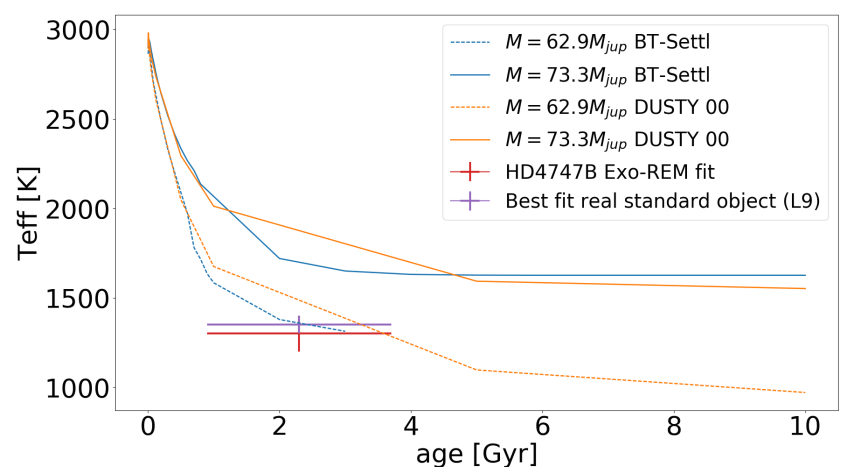

Fig. 14. Comparison between evolutionary models (BT-Settl Allard et al. 2011), real standard objects, and the Exo-REM forward modeling. The predictions from the DUSTY model (Chabrier et al. 2000) are also shown. The evolutionary models seem to underestimate the cooling rate of the substellar objects.

evolutionary models. We used the SPHERE instrument to obtain high-precision astrometric and spectroscopic measurements to refine its measured parameters and allow a more thorough comparison with models.

The HD 4747AB system was analyzed by combining radial velocity measurements and high-contrast imaging. With a spectroscopic analysis we derived an effective temperature $T_{\text {eff }}=$ $5400 \pm 60 \mathrm{~K}$, a $\log (g)=4.60 \pm 0.15$, and a metallicity $[\mathrm{Fe} / \mathrm{H}]=-0.23 \pm 0.05$ dex for the primary star. A primary mass of $M_{\text {star }}=0.856 \pm 0.014 M_{\odot}$ and an age of $2.3 \pm 1.4$ Gyr were also derived. Combining the SPHERE data with new radial velocity measurements from the CORALIE spectrograph, and a detection in an archival NACO dataset with previously published epochs, we derived a dynamical mass of $m_{\mathrm{B}}=70.2 \pm 1.6 M_{\text {Jup }}$.

We adjusted the spectrum extracted from the SPHERE IFS and IRDIS data with known standard objects and derived an L9 spectral type, which is in good agreement with our color-magnitude diagram derived from the IRDIS $K_{1}$ and $K_{2}$ filters and previous observations. A forward analysis was conducted by using Exo-REM and confirmed an effective temperature of $T_{\text {eff }}=1300 \pm 100 \mathrm{~K}$ and a cloudy atmosphere. A radius $R=0.91 \pm 0.16 R_{\text {Jup }}$ has been derived; however, the $\log (g)=$ $4 \pm 0.5$ found does not reproduce the dynamical mass derived in this study. HD 4747B is the most massive object analyzed with Exo-REM, and more work is needed to investigate why the gravity is apparently underestimated with this model.

A retrieval analysis allowed to derive the chemistry of the atmosphere, the temperature-pressure profile, and the carbon and oxygen abundances $\left(\mathrm{C} / \mathrm{H}=-4.72_{-0.39}^{+0.47}, \mathrm{O} / \mathrm{H}=-3.79_{-0.08}^{+0.16}\right)$. We compared these values to the HR 8799 planets and show that a formation scenario for HD 4747B by gravitational instability is compatible, which is favored as well by the mass ratio between the primary and its companion.

Finally a comparison with the BT-Settl evolutionary models was conducted. The effective temperature and $\log (g)$ derived are in good agreement with the spectral analysis in this paper. By comparing the age and the effective temperature, we obtain a model-dependent mass estimation around $65 M_{\mathrm{Jup}}$, which slightly differs from our dynamical estimation.

Acknowledgements. This work has been carried out within the frame of the National Center for Competence in Research "PlanetS" supported by the Swiss National Science Foundation (SNSF). SPHERE is an instrument desigend and built by a consortium consisting of IPAG (Grenoble, France), MPIA (Heidelberg, Germany), LAM (Marseille, France), LESIA (Paris, France), Laboratoire Lagrange (Nice, France), INAF - Osservatorio di Padova (Italy), Observatoire Astronomique de l'Université de Genève (Switzerland), ETH 
Zurich (Switzerland), NOVA (Netherlands), ON - ERA (France), and ASTRON (Netherlands) in collaboration with ESO. SPHERE was funded by ESO, with additional contributions from CNRS (France), MPIA (Germany), INAF (Italy), FINES (Switzerland), and NOVA (Netherlands). SPHERE also received funding from the European Commission Sixth and Seventh Framework Programmes as part of the Optical Infrared Coordination Network for Astronomy (OPTICON) under grant number RII3-Ct-2004-001566 for FP6 (2004-2008), grant number 226604 for FP7 (2009-2012), and grant number 312430 for FP7 (2013-2016). This publication makes use of The Data \& Analysis Center for Exoplanets (DACE), which is a facility based at the University of Geneva $(\mathrm{CH})$ dedicated to extrasolar planets data visualization, exchange, and analysis. DACE is a platform of the Swiss National Centre of Competence in Research (NCCR) PlanetS, federating the Swiss expertise in Exoplanet research. The DACE platform is available at https://dace.unige.ch. This publication makes use of data product from the Two Micron All Sky Survey, which is a joint project of the University of Massachusetts and the Infrared Processing and Analysis Center/California Institute of Technology, funded by the National Aeronautics and Space Administration and the National Science Foundation. This research has benefitted from the SpeX Prism Spectral Libraries, maintained by Adam Burgasser at http://pono.ucsd.edu/ adam/browndwarfs/spexprism. This publication makes use of VOSA, developed under the Spanish Virtual Observatory project supported from the Spanish MICINN through grant AyA2011-24052. J.L.B. acknowledges the support of the UK Science and Technology Facilities Council. Finally, we acknowledge support from the "Progetti Premiali" funding scheme of the Italian Ministry of Education, University, and Research.

\section{References}

Allard, F., Homeier, D., \& Freytag, B. 2011, in 16th Cambridge Workshop on Cool Stars, Stellar Systems, and the Sun, eds. C. Johns-Krull, M. K. Browning, \& A. A. West, ASP Conf. Ser., 448,91

Allard, F., Homeier, D., \& Freytag, B. 2012, Phil. Trans. Royal Soc. London, Ser A, 370, 2765

Amarsi, A. M., Asplund, M., Collet, R., \& Leenaarts, J. 2015, MNRAS, 454, L11

Anderson, D. R., Collier Cameron, A., Hellier, C., et al. 2011, ApJ, 726, L19

Baraffe, I., Chabrier, G., Barman, T. S., Allard, F., \& Hauschildt, P. H. 2003, A\&A, 402, 701

Baraffe, I., Homeier, D., Allard, F., \& Chabrier, G. 2015, A\&A, 577, A42

Baudino, J.-L., Bézard, B., Boccaletti, A., et al. 2015, A\&A, 582, A83

Baudino, J.-L., Molliere, P., Venot, O., et al. 2017, ApJ, 850, 150

Bayliss, D., Hojjatpanah, S., Santerne, A., et al. 2017, AJ, 153, 15

Bayo, A., Rodrigo, C., Barrado, Y., Navascués, D., et al. 2008, A\&A, 492, 277

Bensby, T., Feltzing, S., \& Oey, M. S. 2014, A\&A, 562, A71

Beuzit, J.-L., Feldt, M., Dohlen, K., et al. 2008, in Ground-based and Airborne Instrumentation for Astronomy II, Proc. SPIE, 7014, 701418

Biller, B. A., Liu, M. C., Wahhaj, Z., et al. 2010, ApJ, 720, L82

Boccaletti, A., Riaud, P., Baudoz, P., et al. 2004, PASP, 116, 1061

Bonnefoy, M., Zurlo, A., Baudino, J. L., et al. 2016, A\&A, 587, A58

Brewer, J. M., Fischer, D. A., Valenti, J. A., \& Piskunov, N. 2016, ApJS, 225, 32

Burgasser, A. J. 2007a, ApJ, 659, 655

Burgasser, A. J. 2007b, ApJ, 658, 617

Burgasser, A. J. 2014, ASI Conf. Ser., 11, 7

Burgasser, A. J., Geballe, T. R., Leggett, S. K., Kirkpatrick, J. D., \& Golimowski, D. A. 2006, ApJ, 637, 1067

Burgasser, A. J., Aganze, C., Escala, I., et al. 2016, AAS Meeting Abstracts, 227, 434.08

Burningham, B., Cardoso, C. V., Smith, L., et al. 2013, MNRAS, 433, 457

Burrows, A., Marley, M., Hubbard, W. B., et al. 1997, ApJ, 491, 856

Burrows, A., Heng, K., \& Nampaisarn, T. 2011, ApJ, 736, 47

Butler, R. P., Vogt, S. S., Laughlin, G., et al. 2017, AJ, 153, 208

Casagrande, L., Schönrich, R., Asplund, M., et al. 2011, A\&A, 530, A138

Chabrier, G., \& Baraffe, I. 2000, ARA\&A, 38, 337

Chabrier, G., Baraffe, I., Allard, F., \& Hauschildt, P. 2000, ApJ, 542, 464

Chauvin, G., Lagrange, A.-M., Bonavita, M., et al. 2010, A\&A, 509, A52

Claudi, R. U., Turatto, M., Gratton, R. G., et al. 2008, in Ground-based and Airborne Instrumentation for Astronomy II, Proc. SPIE, 7014, 70143E

Crepp, J. R., Gonzales, E. J., Bechter, E. B., et al. 2016, ApJ, 831, 136

Crepp, J. R., Principe, D. A., Wolff, S., et al. 2018, ApJ, 853, 192

da Silva, L., Girardi, L., Pasquini, L., et al. 2006, A\&A, 458, 609

Desidera, S., Covino, E., Messina, S., et al. 2015, A\&A, 573, A126

Dohlen, K., Langlois, M., Saisse, M., et al. 2008, in Ground-based and Airborne Instrumentation for Astronomy II, Proc. SPIE, 7014, 70143L

D’Orazi, V., Biazzo, K., Desidera, S., et al. 2012, MNRAS, 423, 2789

D’Orazi, V., Desidera, S., Gratton, R. G., et al. 2017, A\&A, 598, A19
Dupuy, T. J., \& Liu, M. C. 2017, ApJS, 231, 15

Ehrenreich, D., Lagrange, A.-M., Montagnier, G., et al. 2010, A\&A, 523, A73

Famaey, B., Jorissen, A., Luri, X., et al. 2005, A\&A, 430, 165

Foreman-Mackey, D., Hogg, D. W., Lang, D., \& Goodman, J. 2013, PASP, 125, 306

Fuhrmann, K., Chini, R., Kaderhandt, L., \& Chen, Z. 2017, ApJ, 836, 139

Fusco, T., Sauvage, J.-F., Petit, C., et al. 2014, in Adaptive Optics Systems IV, Proc. SPIE, 9148, 91481U

Gaia Collaboration (Brown, A. G. A., et al.) 2016, A\&A, 595, A2

Galland, F., Lagrange, A.-M., Udry, S., et al. 2005, A\&A, 443, 337

Gáspár, A., Rieke, G. H., \& Balog, Z. 2013, ApJ, 768, 25

Goodman, J., \& Weare, J. 2010, Commun. Appl. Math. Comput. Sci., 5, 65

Grether, D., \& Lineweaver, C. H. 2006, ApJ, 640, 1051

Hagelberg, J., Ségransan, D., Udry, S., \& Wildi, F. 2016, MNRAS, 455, 2178

Halbwachs, J. L., Arenou, F., Mayor, M., Udry, S., \& Queloz, D. 2000, A\&A, 355,581

Helling, C., \& Casewell, S. 2014, A\&ARv, 22, 80

Katsova, M. M., \& Livshits, M. A. 2011, Astron. Rep., 55, 1123

Kaufer, A., Wolf, B., Andersen, J., \& Pasquini, L. 1997, The Messenger, 89, 1

King, R. R., McCaughrean, M. J., Homeier, D., et al. 2010, A\&A, 510, A99

Kirkpatrick, J. D. 2005, ARA\&A, 43, 195

Konopacky, Q. M., Ghez, A. M., Barman, T. S., et al. 2010, ApJ, 711, 1087

Langlois, M., Vigan, A., Moutou, C., et al. 2013, in Proc. Third AO4ELT Conference, eds. S. Esposito, \& L. Fini, 63

Lavie, B., Mendonça, J. M., Mordasini, C., et al. 2017, AJ, 154, 91

Lee, J.-M., Heng, K., \& Irwin, P. G. J. 2013, ApJ, 778, 97

Line, M. R., Teske, J., Burningham, B., Fortney, J. J., \& Marley, M. S. 2015, ApJ, 807, 183

Looper, D. L., Kirkpatrick, J. D., \& Burgasser, A. J. 2007, AJ, 134, 1162

Maire, A.-L., Boccaletti, A., Rameau, J., et al. 2014, A\&A, 566, A126

Maire, A.-L., Bonnefoy, M., Ginski, C., et al. 2016a, A\&A, 587, A56

Maire, A.-L., Langlois, M., Dohlen, K., et al. 2016b, in Ground-based and Airborne Instrumentation for Astronomy VI, Proc. SPIE, 9908, 990834

Mamajek, E. E., \& Hillenbrand, L. A. 2008, ApJ, 687, 1264

Marcy, G. W., \& Butler, R. P. 2000, PASP, 112, 137

Marois, C., Correia, C., Véran, J.-P., \& Currie, T. 2014, IAU Symp., 299, 48

Mortier, A., Santos, N. C., Sousa, S., et al. 2013, A\&A, 551, A112

Nakajima, T., Oppenheimer, B. R., Kulkarni, S. R., et al. 1995, Nature, 378, 463

Nidever, D. L., Marcy, G. W., Butler, R. P., Fischer, D. A., \& Vogt, S. S. 2002, ApJS, 141, 503

Nordström, B., Mayor, M., Andersen, J., et al. 2004, A\&A, 418, 989

Öberg, K. I., Murray-Clay, R., \& Bergin, E. A. 2011, ApJ, 743, L16

Oppenheimer, B. R., Kulkarni, S. R., Matthews, K., \& Nakajima, T. 1995, Science, 270, 1478

Pavlov, A., Möller-Nilsson, O., Feldt, M., et al. 2008, in Advanced Software and Control for Astronomy II, Proc. SPIE, 7019, 701939

Pinfield, D. J., Burningham, B., Lodieu, N., et al. 2012, MNRAS, 422, 1922

Queloz, D., Mayor, M., Naef, D., et al. 2000, in From Extrasolar Planets to Cosmology: The VLT Opening Symposium, eds. J. Bergeron, \& A. Renzini (Berlin: Springer), 548

Rebolo, R., Zapatero Osorio, M. R., \& Martín, E. L. 1995, Nature, 377, 129

Sahlmann, J., Lovis, C., Queloz, D., \& Ségransan, D. 2011a, A\&A, 528, L8

Sahlmann, J., Ségransan, D., Queloz, D., et al. 2011b, A\&A, 525, A95

Santos, N. C., Israelian, G., Mayor, M., et al. 2005, A\&A, 437, 1127

Siverd, R. J., Beatty, T. G., Pepper, J., et al. 2012, ApJ, 761, 123

Skilling, J. 2006, Bayesian Anal., 1, 833

Sokal, A. 1997, Monte Carlo Methods in Statistical Mechanics: Foundations and New Algorithms, eds. C. DeWitt-Morette, P. Cartier, \& A. Folacci (Boston, MA: Springer US), 131

Thalmann, C., Carson, J., Janson, M., et al. 2009, ApJ, 707, L123

Trotta, R. 2008, Contemp. Phys., 49, 71

Troup, N. W., Nidever, D. L., De Lee, N., et al. 2016, AJ, 151, 85

Valenti, J. A., \& Fischer, D. A. 2005, ApJS, 159, 141

van Leeuwen, F. 2007, A\&A, 474, 653

Vigan, A., Moutou, C., Langlois, M., et al. 2010, in Ground-based and Airborne Instrumentation for Astronomy III, Proc. SPIE, 7735, 77352X

Vigan, A., Gry, C., Salter, G., et al. 2015, MNRAS, 454, 129

Vigan, A., Bonnefoy, M., Ginski, C., et al. 2016, A\&A, 587, A55

Vogt, S. S., Allen, S. L., Bigelow, B. C., et al. 1994, in Instrumentation in Astronomy VIII, eds. D. L. Crawford, \& E. R. Craine, Proc. SPIE, 2198, 362

Wilson, P. A., Hébrard, G., Santos, N. C., et al. 2016, A\&A, 588, A144

Zechmeister, M., \& Kürster, M. 2009, A\&A, 496, 577

Zurlo, A., Vigan, A., Galicher, R., et al. 2016, A\&A, 587, A57 


\section{Appendix A: Archival NACO observation: $\chi^{2}$ maps over double roll subtraction angles}

As described in Sect. 4.3, we computed the $\chi^{2}$ maps for each double roll subtraction angle from $5^{\circ}$ to $25^{\circ}$. Each map was computed from 20 to 55 pixel of separation $\left(346^{\prime \prime}-953^{\prime \prime}\right)$ and from $90^{\circ}$ to $270^{\circ}$, the rest of the image being excluded by the orbital information from the epochs from Crepp et al. (2016), our SPHERE observation and the radial velocities. In Fig. A.1 we show these maps and the median over the double roll subtraction angles (Fig. A.1f). The position of the companion is retrieved as the minimum $\chi^{2}$ on each map, except for an angle of $20^{\circ}$ where another minimum is detected. However a local minimum is clearly identified at the companion position; when the angle increases there is less signal on the companion. By construction, a larger double roll subtraction angle means fewer images to add together, and thus less signal for the detection.

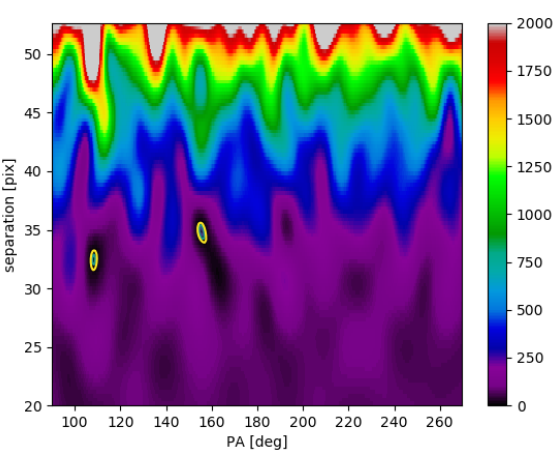

(a)

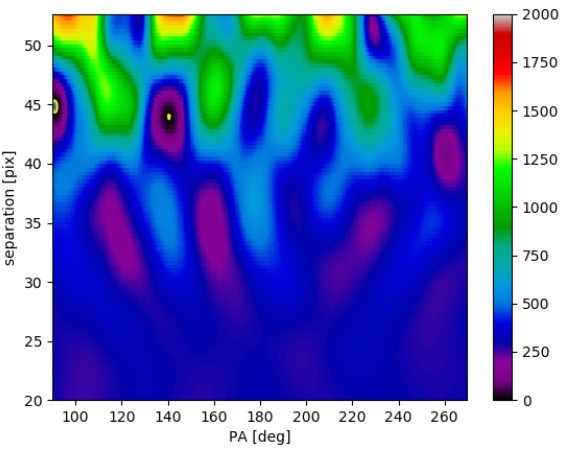

(d)

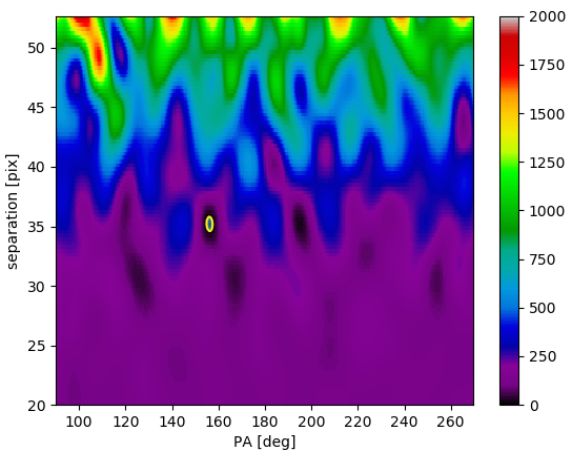

(b)

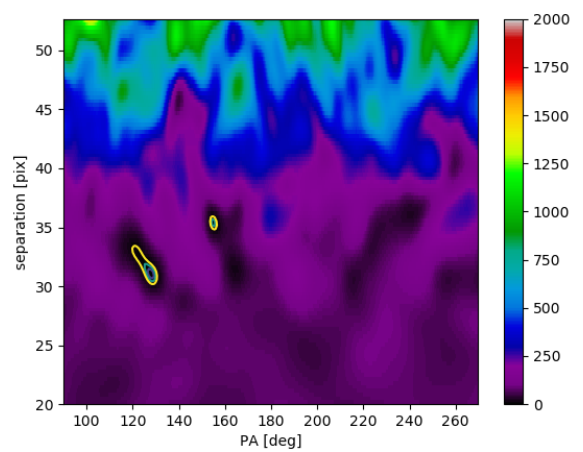

(e)

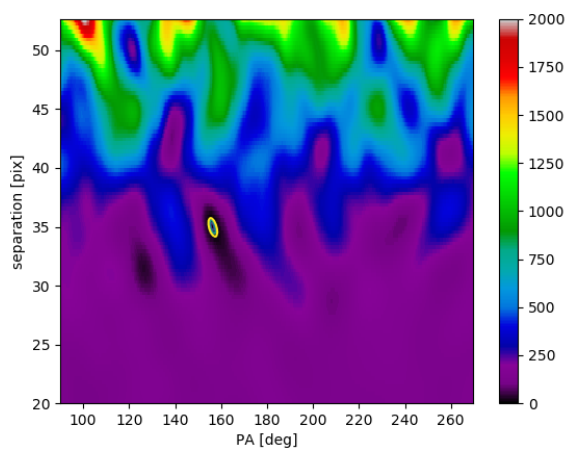

(c)

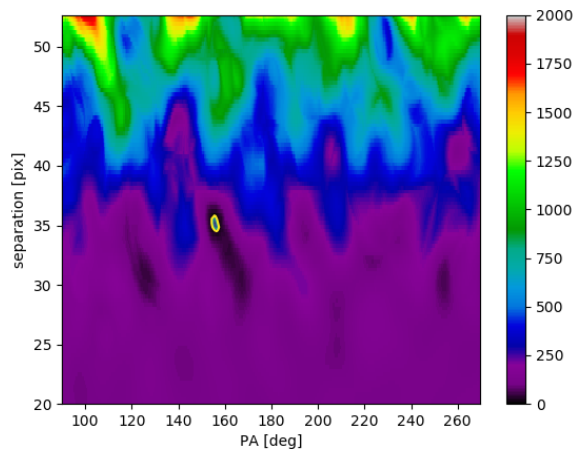

(f)

Fig. A.1. Archival NACO data reduction. A $\chi^{2}$ map of the image for each double roll subtraction angle. The maps shown have been computed for position angles between $90^{\circ}$ and $270^{\circ}$ and separations between 20 and 50 pixels as the companion is completely excluded in the rest of the image by the orbital analysis from the RV, Keck, and SPHERE observations. Panel $a$ : $\chi^{2}$ map of the image with a double roll subtraction angle of $5^{\circ}$. Panel $b: \chi^{2}$ map of the image with a double roll subtraction angle of $10^{\circ}$. Panel $c: \chi^{2}$ map of the image with a double roll subtraction angle of $15^{\circ}$. Panel $d: \chi^{2}$ map of the image with a double roll subtraction angle of $20^{\circ}$. Panel $e: \chi^{2}$ map of the image with a double roll subtraction angle of $25^{\circ}$. Panel $f$ : median $\chi^{2}$ map over double roll subtraction angles. The minimum shows the position of the companion. 


\section{Appendix B: MCMC orbital parameters}

The MCMC simulation was performed using emcee (ForemanMackey et al. 2013), a python stable implementation of the affine-invariant ensemble sampler for MCMC proposed by Goodman \& Weare (2010). The data are modeled with a Keplerian and four RV offsets (one for HIRES, and three for the different versions of CORALIE: C98, C07, C14). The noise in the radial velocity data is modeled with a nuisance parameter for each instrument. The parallax and the mass of the primary star are also parameters of the MCMC. We ran the MCMC simulation with 39 walkers and $10^{6}$ steps for each walker. We then computed the correlation timescale $\tau$ of each walker and got rid of the initialization bias by removing the first $20 \tau$ for each one (Sokal 1997). To build a statistically meaningful sample, we then sampled each walker by its coherence timescale.

At the end we have 358000 independent data points, which means that we characterize the parameters at a $1 / \sqrt{358000}=$ $0.17 \%$ accuracy which corresponds to a $3 \sigma$ confidence interval.

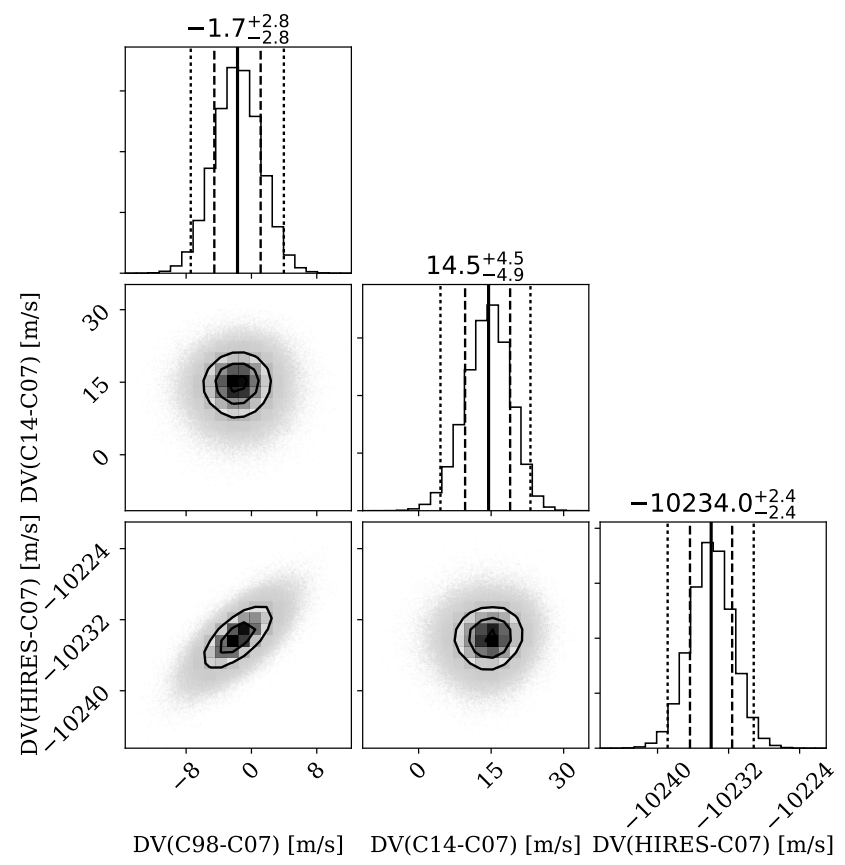

Fig. B.1. Marginalized 1D and 2D posterior distributions of the orbital global adjustment combining radial velocities and direct imaging data. Shown are the offsets between the different radial velocity instruments. C98 stands for the first version of the CORALIE instrument, C07 for its first update in 2007, and C14 for the last one in 2014. 
S. Peretti et al.: Orbital and spectral analysis of the benchmark brown dwarf HD 4747B

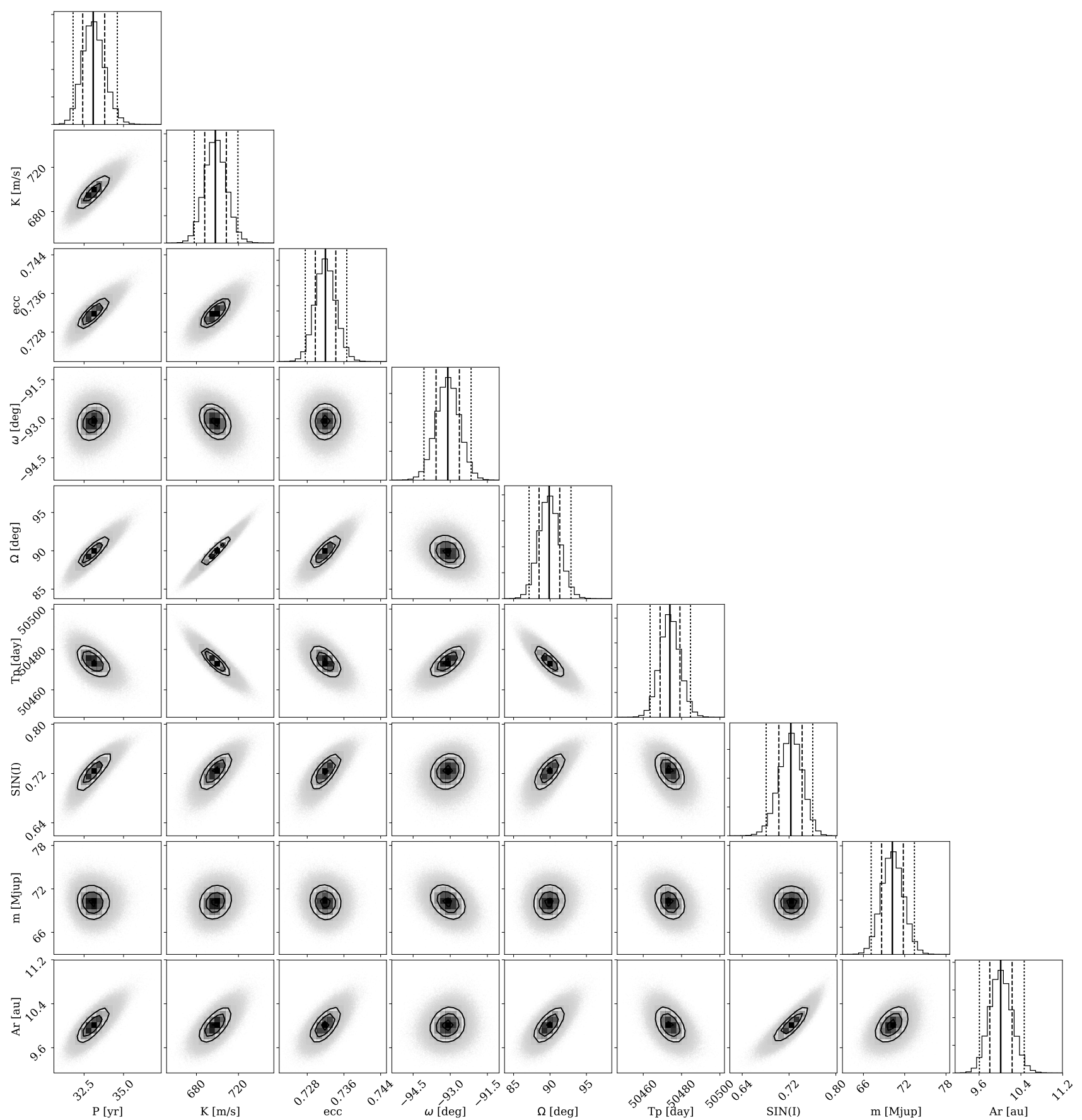

Fig. B.2. Marginalized 1D and 2D posterior distributions of the orbital global adjustment combining radial velocities and direct imaging data. The model parameters $P, K, e, \omega, \Omega, T_{\mathrm{p}}$, and $\sin (i)$ are shown, as are the derived quantities, the mass (m), and the orbital semi major axis (Ar). 


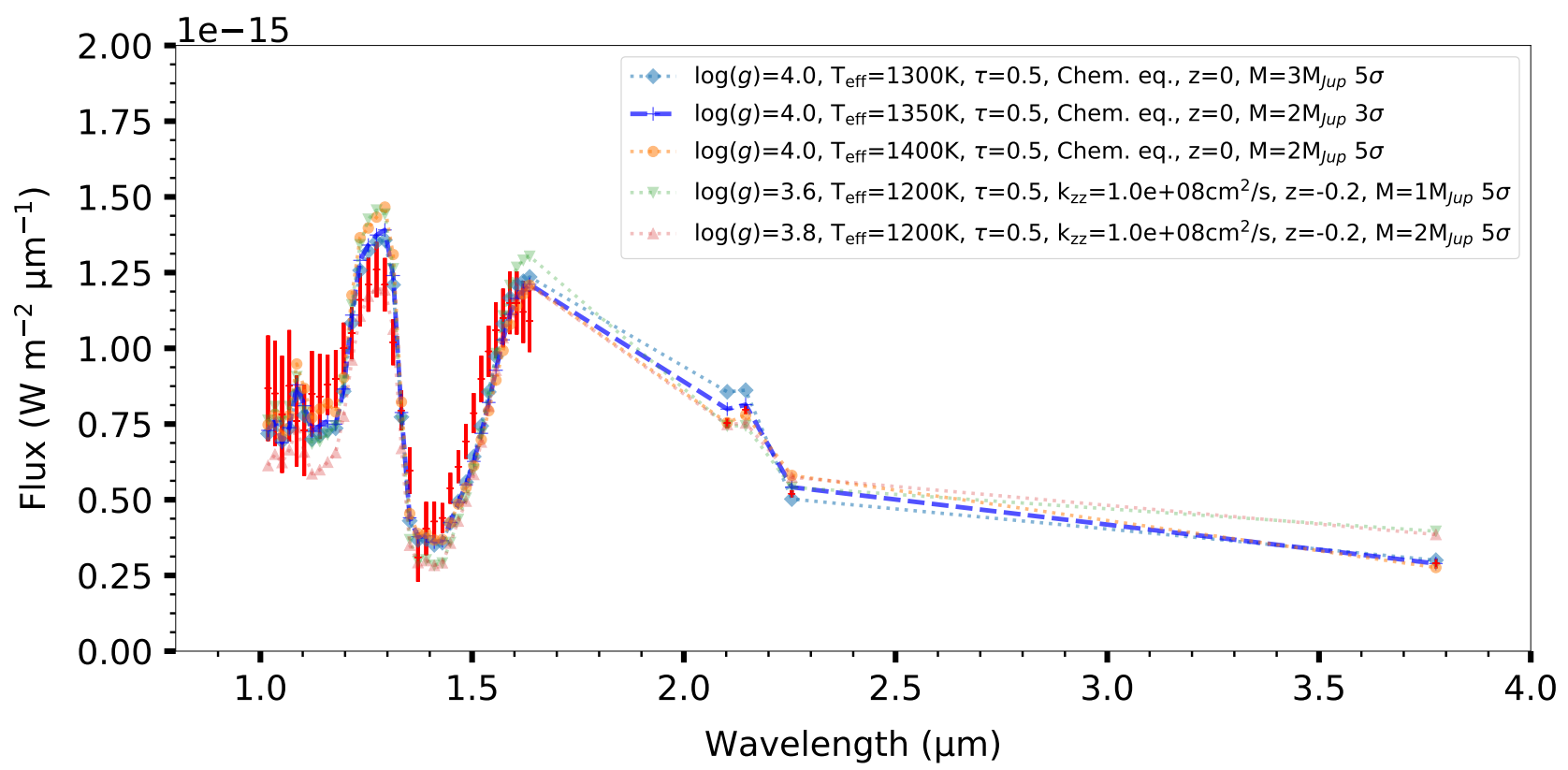

Fig. B.3. Exo-Rem best fit to spectrum. The data are represented in red and the best fit is shown by the light blue dashed line.

\section{Appendix C: Retrieval of additional results}

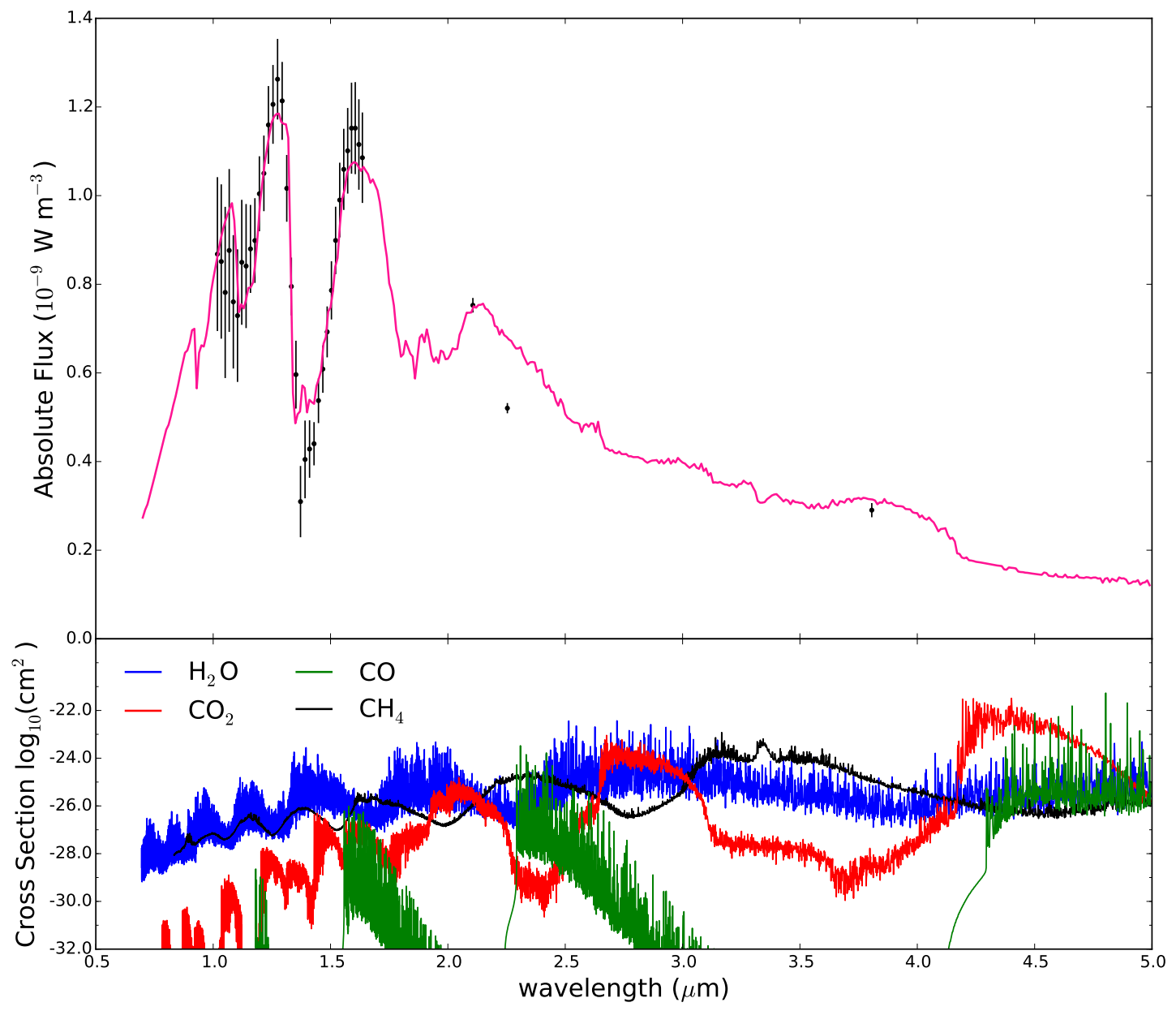

Fig. C.1. Best-fit spectra obtained with HELIOS-R (top panel) and cross-section of the four main absorbers (bottom panel) at a pressure of 0.1 bar and a temperature of $1900 \mathrm{~K}$. The lack of observations in the $K$ band block the detection of $\mathrm{CO}$ (CO absorption band between 1.5 and $2 \mu \mathrm{m}$ ). 


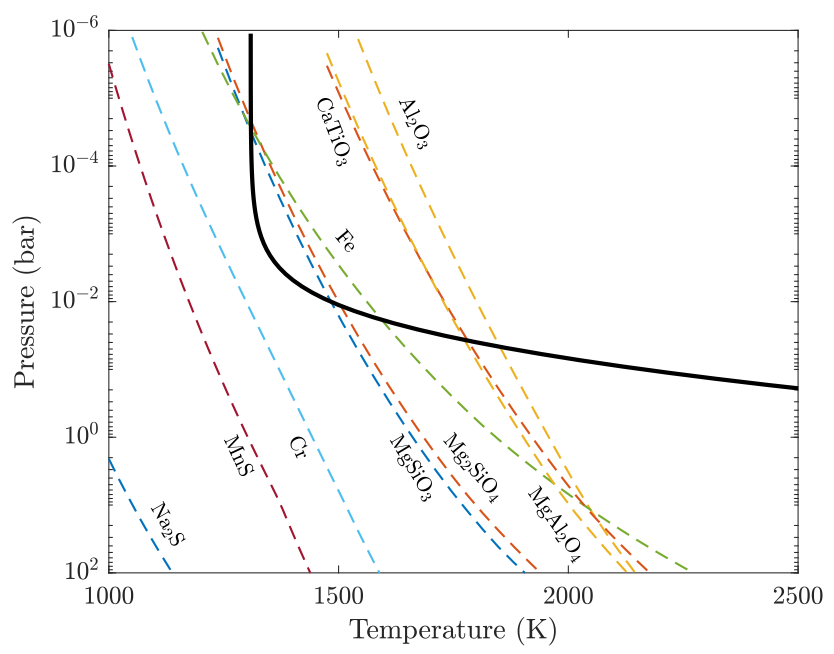

Fig. C.2. Temperature-pressure profiles for HD 4747B obtained with HELIOS-R. Dashed lines are condensation curves for some possible condensates (assuming solar composition).

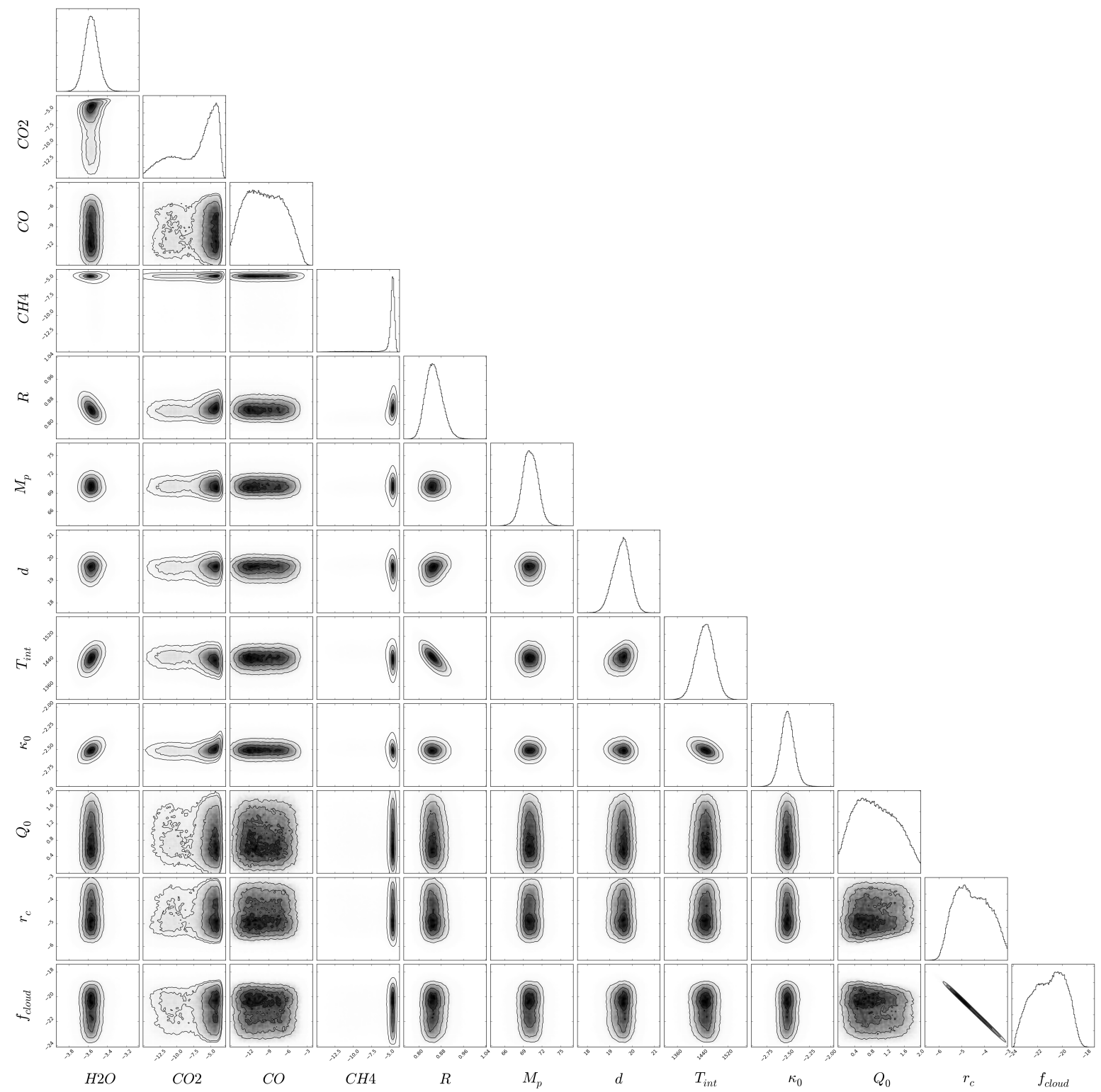

Fig. C.3. Results of the retrieval. Montage of posterior distributions from the best model (unconstrained chemistry with clouds) of HD 4747B. 

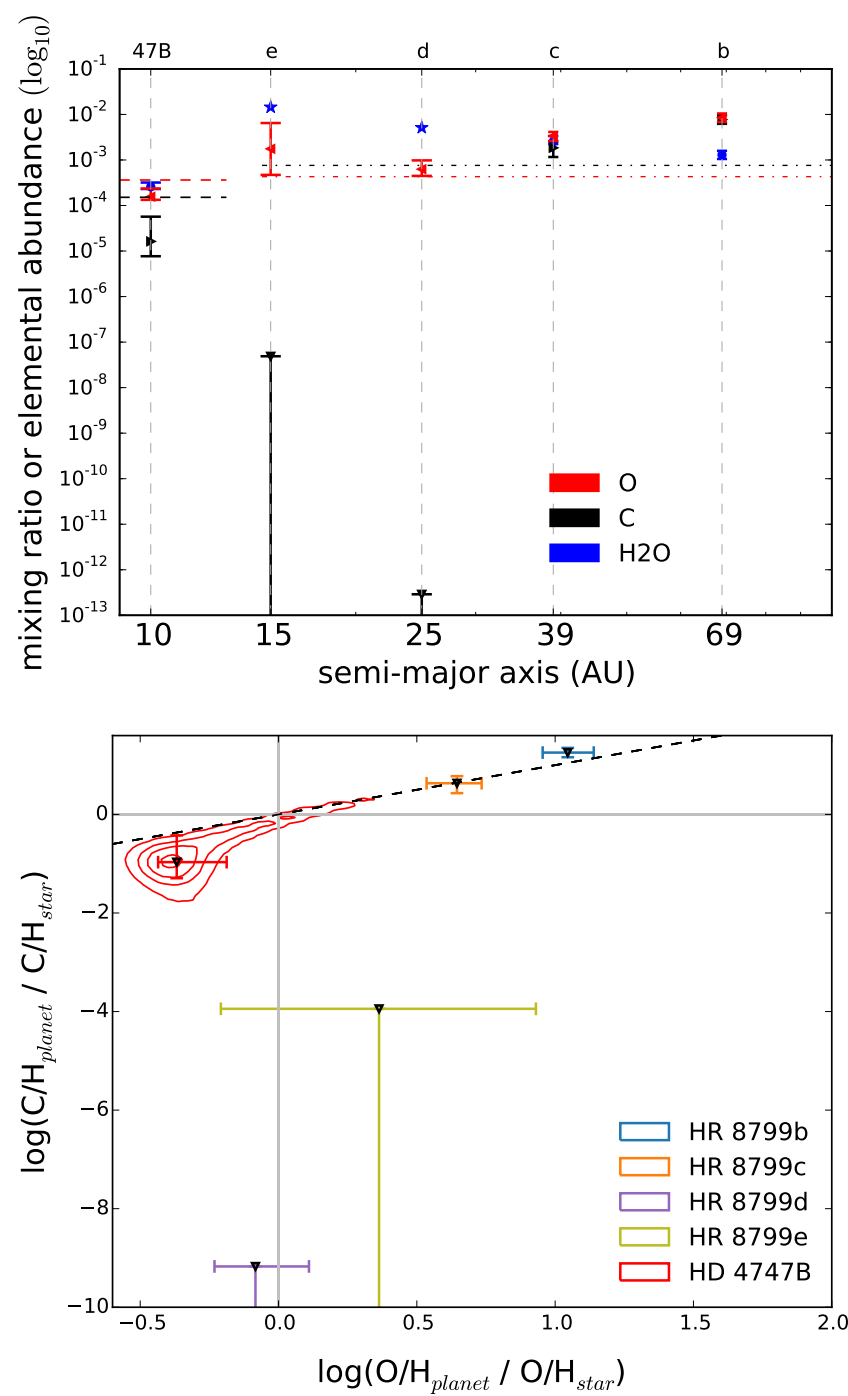

Fig. C.4. Summary of our main results. Same as Fig. 12, but with HR 8799e and HR 8799d. Top panel: retrieved water mixing ratios and elemental abundances of carbon and oxygen for HD 4747B and for the four HR 8799 exoplanets as a function of the distance to the host star. For HR 8799d and e, we show the water abundance in chemical equilibrium at 1 bar (represented by the blue stars). The carbon and oxygen abundances of the stars are shown with the dashed lines (HD 4747) and dashed dot lines(HR 8799). Bottom panel: companion elemental abundances normalized to its stellar values with the dashed black line denoting parity. 